\title{
L-embedded Banach spaces and measure topology
}

\section{H. Pfitzner}

\begin{abstract}
An L-embedded Banach spaace is a Banach space which is complemented in its bidual such that the norm is additive between the two complementary parts. On such spaces we define a topology, called an abstract measure topology, which by known results coincides with the usual measure topology on preduals of finite von Neumann algebras (like $\left.\mathrm{L}^{1}([0,1])\right)$. Though not numerous, the known properties of this topology suffice to generalize several results on subspaces of $\mathrm{L}^{1}([0,1])$ to subspaces of arbitrary L-embedded spaces.
\end{abstract}

\section{$\S 1$ Introduction}

This article continues the investigations made in [23, 24] on asymptotically isometric copies of $l^{1}$ in preduals of von Neumann algebras and in L-embedded Banach spaces. (For defintions see below.) In 24] it has been proved that, roughly speaking, in the predual of a finite von Neumann algebra the only non-trivial bounded sequences that converge to 0 with respect to the measure topology are essentially those that span $l^{1}$ asymptotically; for $\mathrm{L}^{1}(\mu), \mu$ a finite measure, this characterization has been known for quite a time 15, Th. 2], [25, Th. 3, Rem. 6bis].

From the point of view of Banach space theory, L-embedded Banach spaces provide a natural frame for preduals of von Neumann algebras. So the starting point of this paper is on the one hand the definition of an abstract measure topology, Definition 3, patterned after the just mentionend characterization and on the other hand the easy but important observation, Theorem 1 , that every L-embedded space admits such a topology. Although this topology does not come out easily with its properties - at the time of this writing it is not clear whether it is Hausdorff let alone metrizable or whether addition is continuous - it allows to generalize several results on subspaces of $\mathrm{L}^{1}(\mu)$ to subspaces of arbitrary L-embedded spaces. Thus section $\S 4$ of the present paper is titled "Section IV.3 of [13] (partly) revisited". For example, Theorem 10 generalizes a theorem of Buhvalov-Lozanovskii which describes the link between L-embeddedness and measure topology for subspaces $Y$ of $\mathrm{L}^{1}(\mu), \mu$ finite: $Y$ is L-embedded if and only if its unit ball is closed in measure. (Note in passing that this criterion involves only the space $Y$ itself, not its bidual.) Moreover, as a consequence of this, the closedness in measure of the unit ball of $Y$ is a weak substitute for compactness which could be called "convex sequential compactness", see Corollary 9. We also reprove a result of Godefroy and Li concerning a criterion for L-embedded subspaces which are duals of M-embedded spaces, see Theorem 13 . In this vein, that is by substituting arbitrary L-embedded spaces for $\mathrm{L}^{1}(\mu)$, we recover also some results of Godefroy, Kalton, Li [10] in $\S 5$. Finally, in $\S 6$ it is proved that addition is $\tau_{\mu}$-continuous in preduals of von Neumann algebras.

\section{$\S 2$ Notation, Background:}

The results are stated for complex scalars. The dual of a Banach space $X$ is denoted by $X^{\prime}$. $\mathrm{B}_{X}$ denotes the unit ball of $X$. Subspace of a Banach space means norm-closed subspace, bounded always means normbounded. As usual, we consider a Banach space as a subspace of its bidual omitting the canonical embedding. $\left[x_{n}\right]$ denotes the closed linear span of a (finite or infinite) sequence $\left(x_{n}\right)$.

Basic properties and definitions which are not explained here can be found in [4] or in [20]-21] for Banach spaces and in [22, 28] for $\mathrm{C}^{*}$-algebras. The standard reference for $\mathrm{M}$ - and L-embedded spaces is the monograph [13].

Let $\left(x_{n}\right)$ be a sequence of nonzero elements in a Banach space $X$.

We say that $\left(x_{n}\right)$ spans $l^{1}$ isomorphically (or $r$-isomorphically to be more precise) - $\left(x_{n}\right)_{n \in \mathbb{N}} \stackrel{r}{\sim} l^{1}$ or just $x_{n} \stackrel{r}{\sim} l^{1}$ in symbols - if there exists $r>0$ (trivially $r \leq 1$ ) such that $r\left(\sum_{n=1}^{\infty}\left|\alpha_{n}\right|\right) \leq\left\|\sum_{n=1}^{\infty} \alpha_{n} \frac{x_{n}}{\left\|x_{n}\right\|}\right\| \leq$ $\sum_{n=1}^{\infty}\left|\alpha_{n}\right|$ for all scalars $\alpha_{n}$ (the second inequality being trivial).

We say that $\left(x_{n}\right)$ spans $l^{1}$ almost isometrically $-x_{n} \stackrel{\text { alm }}{\sim} l^{1}$ in symbols - if there is a sequence $\left(\delta_{m}\right)$ in $\left[0,1\right.$ [ tending to 0 such that $\left(x_{n}\right)_{n \geq m} \stackrel{1-\delta_{m}}{\sim} l^{1}$ for all $m \in \mathbb{N}$. Recall that the Banach-Mazur distance of two Banach spaces $X$ and $Y$ is defined by $\operatorname{dist}(X, Y)=\inf \|T\|\left\|T^{-1}\right\|$ where the infimum extends over all surjective isomorphisms $T: X \rightarrow Y$. To avoid confusion, notice that $\operatorname{dist}\left(l^{1},\left[x_{n}\right]\right)=1$ is not the same as $x_{n} \stackrel{\text { alm }}{\sim} l^{1}$. 
Finally, a sequence $\left(x_{n}\right)$ is said to span $l^{1}$ asymptotically isometrically or just to span $l^{1}$ asymptotically - $x_{n} \stackrel{\text { asy }}{\sim} l^{1}$ in symbols - if there is a sequence $\left(\delta_{n}\right)$ in $\left[0,1\left[\right.\right.$ tending to 0 such that $\sum_{n=1}^{\infty}\left(1-\delta_{n}\right)\left|\alpha_{n}\right| \leq$ $\left\|\sum_{n=1}^{\infty} \alpha_{n} \frac{x_{n}}{\left\|x_{n}\right\|}\right\| \leq \sum_{n=1}^{\infty}\left|\alpha_{n}\right|$ for all scalars $\alpha_{n}$.

Note that the present definitions of almost and asymptotically isometric differ slightly from those in [6], [23] by the term $x_{n} /\left\|x_{n}\right\|$ but that, of course, for normalized sequences the definitions are the same. Note also the technical detail that because of this term one might have $\left\|x_{n}\right\| \rightarrow 0$ for a sequence spanning $l^{1}$ isomorphically whereas sequences that are equivalent to the canonical $l^{1}$-basis ([4, p. 43]) are uniformly bounded away from 0 . We say that a Banach space is isomorphic (respectively almost isometric respectively asymptotically isometric) to $l^{1}$ if it has a basis with the corresponding property.

Let $Y$ be a subspace of a Banach space $X$ and $P$ be a projection on $X . P$ is called an $L$-projection provided $\|x\|=\|P x\|+\left\|\left(\mathrm{id}_{X}-P\right) x\right\|$ for all $x \in X$. A subspace $Y \subset X$ is called an $M$-ideal in $X$ if its annihilator $Y^{\perp}$ in $X^{\prime}$ is the range of an L-projection on $X^{\prime} . Y$ is called an $L$-summand in $X$ if it is the range of an L-projection on $X$. In the special case in which $X=Y^{\prime \prime}$ and in which $Y$ is an M-summand (respectively an L-summand) in $Y^{\prime \prime}$ we say that $Y$ is $M$-embedded (respectively $L$-embedded). As examples we only mention that preduals of von Neumann algebras, in particular $l^{1}$ and $L^{1}$-spaces, furthermore the Hardy space $H_{0}^{1}$ and the dual of the disc algebra are L-embedded. The sequence space $c_{0}$, the space of compact operators on a Hilbert space, and the quotient $C / A$ of the continuous functions on the unit circle by the disc algebra $A$ are examples among M-embedded spaces. It is not difficult but important to see that if there is an L-projection $P$ on a Banach space $X$ then each contractive projection on $X$ which has the same kernel as $P$ coincides with $P$, see [13, Prop. I.1.2]. It follows that if $X$ is M-embedded then the canonical inclusion of $X^{\prime}$ in $X^{\prime \prime \prime}$ is an L-summand in $X^{\prime \prime \prime}$ that is $X^{\prime}$ is L-embedded; the converse is false [13, III.1.3]; in fact, for an L-embedded space being the dual of an M-embedded space can be quite a restrictive condition: For instance, while the dual of any $\mathrm{C}^{*}$-algebra is L-embedded only those $\mathrm{C}^{*}$-algebras are M-embedded which are isometrically *-isomorphic to the algebra of compact operators or to a $c_{0}$-sum of such algebras [13, III.2.9]. Throughout this note, if $X$ denotes an L-embedded Banach space (which is not always the case) we will write $X_{s}$ for the complement of (the canonical embedding of) $X$ in $X^{\prime \prime}$ that is $X^{\prime \prime}=X \oplus_{1} X_{s}$. In this case $P$ will denote the L-projection from $X^{\prime \prime}$ onto $X$.

We recall Godefroy's fundamental result [9], [13, IV.2.2] that L-embedded Banach spaces are $w$-sequentially complete. This will be used mostly without reference; together with Rosenthal's $l^{1}$-theorem a typical application is that each bounded sequence in an L-embedded space contains a subsequence which either spans $l^{1}$ or converges weakly. There is a useful criterion for L-embeddedness of subsapces of L-embedded spaces due to Li ([19] or [13, Th. IV.1.2]) which we state for easy reference:

Lemma 1 (Li) For an L-embedded Banach space $X$ (with L-decomposition $X^{\prime \prime}=X \oplus_{1} X_{s}$ and L-projection $P$ on $X^{\prime \prime}$ with range $\left.X\right)$ and a closed subspace $Y$ of $X$ the following assertions are equivalent.

(i) $Y$ is L-embedded.

(ii) $Y^{\perp \perp}=Y \oplus_{1}\left(Y^{\perp \perp} \cap X_{s}\right)$.

(iii) $P \overline{\mathrm{B}_{Y}} w^{*}=\mathrm{B}_{Y}$.

(iv) $P Y^{\perp \perp}=Y$.

In particular if $Y$ is L-embedded and if one identifies $Y^{\prime \prime}=Y \oplus_{1} Y_{s}$ and $Y^{\perp \perp} \subset X^{\prime \prime}$ then $Y_{s}=Y^{\perp \perp} \cap X_{s}$.

Let us finally cite some technical results from [23] which will be used in the sequel.

It is routine to show that sequences spanning $l^{1}$ asymptotically are stable by adding norm-null sequences [24. Lem. 4], to be more precise, let $\left(x_{n}\right),\left(y_{n}\right)$ be two sequences in a Banach space $X$ such that $\left(x_{n}\right)$ spans $l^{1}$ asymptotically, inf $\left\|x_{n}\right\|>0,\left\|y_{n}\right\| \rightarrow 0$ and $x_{n}+y_{n} \neq 0$. Then $\left(x_{n}+y_{n}\right)$ spans $l^{1}$ asymptotically, too.

Although it has been proved in [6] that there are almost isometric $l^{1}$-copies which do not contain asymptotic ones, both notions "coincide up to subsequences" in L-embedded Banach spaces, more precisely, in Lembedded Banach spaces each sequence spanning $l^{1}$ almost isometrically contains a subsequence spanning $l^{1}$ asymptotically [23, Cor. 3].

The following lemma is fundamental for the rest of the paper. It is an immediate consequence of [23] and says that within L-embedded spaces, sequences spanning $l^{1}$ almost or asymptotically isometrically behave like the standard basis of $l^{1}$ as to their $w^{*}$-accumulation points. Mostly it will be used with $M$ being a countable set of normalized elements that $\operatorname{span} l^{1}$ asymptotically.

Lemma 2 Let $X$ be L-embedded (with L-decomposition $X^{\prime \prime}=X \oplus_{1} X_{s}$ ), let $M \subset X$ be a subset of the unit sphere of $X$. Then the following assertions are equivalent. 
(i) $M$ contains a sequence spanning $l^{1}$ asymptotically.

(ii) $M$ admits a $w^{*}$-accumulation point $x_{s} \in X_{s}$ of norm one.

In fact, each $w^{*}$-accumulation point of a sequence spanning $l^{1}$ asymptotically lies in $X_{s}$.

Proof: (i) $\Rightarrow$ (ii) and the second statement: see last paragraph of the proof of [23, Lem. 1]; (ii) $\Rightarrow$ (i): 23, Th. 2]

\section{$\S 3$ Abstract measure topology}

To prepare the definiton of an abstract measure topology we recall some facts about sequential spaces (see for example [8, 1.6-1.7] or [18], cf. also [17]) because the topology will be defined by determining the class of its convergent sequences.

A topological space is called a sequential space if closedness and sequential closedness coincide. A topological space is called a Fréchet space if closure and sequential closure coincide. Clearly first countable spaces are Fréchet spaces and Fréchet spaces are sequential spaces. Recall that a topological space is a $\mathrm{T}_{1}$-space if every one-point set is closed; this happens if each convergent sequence has a unique limit.

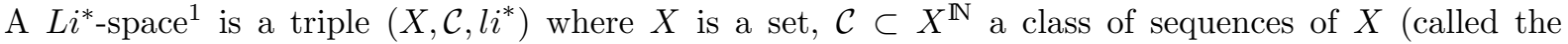
convergence class) and $l i^{*}: \mathcal{C} \rightarrow X$ a map (called limit operator) satisfying the following conditions (L1)(L3). We write $l i^{*} x_{n}$ instead of $l i^{*}\left(\left(x_{n}\right)_{n \in \mathbb{N}}\right)$; the elements of $\mathcal{C}$ are called $\mathcal{C}$-convergent sequences. Let $\left(x_{n}\right)$ be any sequence in $X$, let $x \in X$.

(L1) If $x_{n}=x$ for all $n \in \mathbb{N}$ then $\left(x_{n}\right) \in \mathcal{C}$ and $l i^{*} x_{n}=x$.

(L2) If $\left(x_{n}\right) \in \mathcal{C}$ with $l i^{*} x_{n}=x$ then $\left(x_{n_{k}}\right) \in \mathcal{C}$ and $l i^{*} x_{n_{k}}=x$ for each subsequence $\left(x_{n_{k}}\right)$ of $\left(x_{n}\right)$.

(L3) If a sequence $\left(x_{n}\right)$ is such that there is $x \in X$ and any subsequence $\left(x_{n_{k}}\right)$ contains a further subsequence $\left(x_{n_{k_{m}}}\right)$ such that $\left(x_{n_{k_{m}}}\right) \in \mathcal{C}$ and $l i^{*} x_{n_{k_{m}}}=x$ then $\left(x_{n}\right) \in \mathcal{C}$ and $l i^{*} x_{n}=x$.

On a $L i^{*}$-space $\left(X, \mathcal{C}, l i^{*}\right)$ one defines a topology $\tau_{l i}$ - called the sequential topology induced by $l i^{*}$ - by taking as the family of closed sets all $l i^{*}$-sequentially closed sets; here we call a set $A l i^{*}$-sequentially closed if $l i^{*} x_{n} \in A$ for all $\mathcal{C}$-convergent sequences $\left(x_{n}\right)$ contained in $A$. It is elementary to verify that in this way

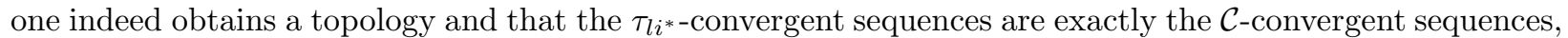
that is for every sequence $\left(x_{n}\right)$ in $X$ one has $l i^{*} x_{n}=x$ if and only if $x_{n} \stackrel{\tau_{l i^{*}}}{\longrightarrow} x$.

An $\mathcal{S}^{*}$-space is a $L i^{*}$-space $\left(X, \mathcal{C}, l i^{*}\right)$ satisfying additionally

(L4) If $\left(x_{m}^{(n)}\right)_{m} \in \mathcal{C}$ for all $n \in \mathbb{N}$ and $\left(x_{n}\right) \in \mathcal{C}$ such that $l i^{*} x_{m}^{(n)}=x_{n}$ for all $n \in \mathbb{N}$ and $l i^{*} x_{n}=x$ then there exist two sequences $\left(n_{k}\right),\left(m_{k}\right)$ such that $l i^{*} x_{m_{k}}^{m_{k}}=x$.

Endowed with $\tau_{l i^{*}}$, an $\mathcal{S}^{*}$-space becomes a Fréchet space [8, 1.7.18,19].

As already mentioned in the introduction the following definition is patterned after a characterization of bounded measure-null sequences in the preduals of finite von Neumann algebras [24. Th. 1].

Definition 3 Let $X$ be a Banach space. A system $\tau_{\mu}$ of subsets of $X$ is called an abstract measure topology if it satisfies the following four conditions.

1. $\left(X, \tau_{\mu}\right)$ is a sequential space in which every convergent sequence has a unique limit.

2. $\tau_{\mu}$ is weaker than the norm topology.

3. $\tau_{\mu}$ is translation invariant for sequences more precisely, $x_{n} \stackrel{\tau_{\mu}}{\rightarrow} x$ if and only if $x_{n}-x \stackrel{\tau_{\mu}}{\rightarrow} 0$ for any sequence $\left(x_{n}\right)$ in $X$.

4. Each bounded sequence in $X$ that spans $l^{1}$ asymptotically $\tau_{\mu}$-converges to 0 , and each sequence in $X$ that $\tau_{\mu}$-converges to 0 is bounded and contains a subsequence which spans $l^{1}$ asymptotically or tends to 0 in norm.

The following result is quite easy to prove. Nevertheless, because of its importance, we call it a theorem.

\footnotetext{
${ }^{1}$ To avgid confusion with the letter L like in L-embedded, L-projection, L-structure we prefer the notation $L i^{*}$ instead of $\mathcal{L}^{*}$ as in [8, 18].
} 
Theorem 4 Every L-embedded Banach space admits an abstract measure topology.

Proof: Let $X$ be an L-embedded Banach space with L-decomposition $X^{\prime \prime}=X \oplus_{1} X_{s}$. Set

$$
\begin{gathered}
\mathcal{C}_{0}=\left\{\left(x_{n}\right) \mid\left(x_{n}\right) \text { is bounded and every subsequence }\left(x_{n_{k}}\right) \text { of }\left(x_{n}\right)\right. \text { contains } \\
\text { a subsequence } \left.\left(x_{n_{k_{l}}}\right) \text { such that } x_{n_{k_{l}}} \stackrel{\text { asy }}{\sim} l^{1} \text { or }\left\|x_{n_{k_{l}}}\right\| \rightarrow 0\right\}, \\
\mathcal{C}=\left\{\left(x_{n}\right) \mid \text { there exists } x \in X \text { such that }\left(x_{n}-x\right) \in \mathcal{C}_{0}\right\} .
\end{gathered}
$$

We define a limit operator $l i^{*}: \mathcal{C} \rightarrow X$ by $l i^{*} x_{n}=x$ where $x \in X$ is such that $\left(x_{n}-x\right) \in \mathcal{C}_{0}$. To show that $\left(X, \mathcal{C}, l i^{*}\right)$ is a $L i^{*}$-space the only thing to verify is that $l i^{*}$ is well defined as a map. because then conditions (L1) - (L3) are immediate from the definiton of $\mathcal{C}$.

Suppose that there are $x, y \in X,\left(x_{n}\right) \in \mathcal{C}$ such that both $\left(x_{n}-x\right) \in \mathcal{C}_{0}$ and $\left(x_{n}-y\right) \in \mathcal{C}_{0}$. If $\left(x_{n}-x\right)$ or $\left(x_{n}-y\right)$ admits a subsequence tending to 0 in norm then $x=y$ because the norm topology is Hausdorff. Otherwise, after passing to an appropriate subsequence, we suppose that both sequences are uniformely bounded away from 0 in norm and that both $x_{n}-x \stackrel{\text { asy }}{\sim} l^{1}$ and $x_{n}-y \stackrel{\text { asy }}{\sim} l^{1}$. Since both sequences are bounded, by Lemma 2 they admit two $w^{*}$-accumulation points $x_{s}, y_{s} \in X_{s}$ and there is a net $\left(x_{n_{\gamma}}\right)$ such that $x_{n_{\gamma}}-x \stackrel{w^{*}}{\rightarrow} x_{s}$ and $x_{n_{\gamma}}-y \stackrel{w^{*}}{\rightarrow} y_{s}$. But this means that $x_{n_{\gamma}} \stackrel{w^{*}}{\rightarrow} x+x_{s}=y+y_{s}$ whence $x=y$ (and $x_{s}=y_{s}$ ).

We define the abstract measure topology $\tau_{\mu}$ as the sequential topology induced by $l i^{*}$. It is immediate from the definition of $\mathcal{C}$ that $\tau_{\mu}$ satisfies the conditions of Definition 3 .

Lemma 5 shows some elementary properties of $\tau_{\mu}$ which in the sequel will be used mostly without reference.

Lemma 5 If a Banach space $X$ admits an abstract measure topology $\tau_{\mu}$ then $\tau_{\mu}$ has the following properties.

(a) $\left(X, \tau_{\mu}\right)$ is a $T_{1}$-space.

(b) The relative topology of $\tau_{\mu}$ on a subspace of $X$ is again an abstract measure topology.

(c) Closedness and sequential closedness coincide for $\tau_{\mu}$. Sequentially continuous maps on $X$ are continuous.

(d) If $X$ does not contain a copy of $l^{1}$ the norm topology is an abstract measure topology and is the only one.

(e) $\tau_{\mu}$ is unique.

(f) If $X$ is the predual of a finite von Neumann algebra then $\tau_{\mu}$ coincides on bounded sets with the usual measure topology; on unbounded sets it does not in general.

(g) Multiplication by scalars is $\tau_{\mu}$-continuous.

(h) If $X$ is L-embedded (i.e. $\left.X^{\prime \prime}=X \oplus_{1} X_{s}\right)$ and if a net $\left(x_{\gamma}\right)$ in $X w^{*}$-converges to $x_{s} \in X_{s}$ such that $\left\|x_{\gamma}\right\| \leq\left\|x_{s}\right\|$ then $x_{\gamma} \stackrel{\tau_{\mu}}{\rightarrow} 0$.

(i) If $X$ is L-embedded then for any $x^{\prime \prime} \in{\overline{\mathrm{B}_{X_{s}}}}^{w^{*}}$ there exists a net $\left(x_{\gamma}\right)$ in $\mathrm{B}_{X}$ such that both $x_{\gamma} \stackrel{\tau_{\mu}}{\rightarrow}$ 0 and $x_{\gamma} \stackrel{w^{*}}{\rightarrow} x^{\prime \prime}$.

Proof: (a) - (d) are clear from the definition.

(e) The topology of sequential spaces is determined by its convergent sequences. Thus the abstract measure topology is unique because the conditions of Definition 3 determine all convergent sequences.

(f) The assertion concerning bounded sets follows from [24, Th. 1]. For the assertion concerning unbounded sets we consider the usual measure (=pointwise) topology and the $w^{*}$-topology on $l^{1}$ : These two and $\tau_{\mu}$ coincide on bounded sets. But the unbounded sequence $\left(n e_{n}\right)$ converges in the usual measure topology while it does not with respect to $\tau_{\mu}$. (Here $\left(e_{n}\right)$ denotes the standard basis of $l^{1}$.)

(g) Let $\lambda_{n} \rightarrow \lambda$ in $\mathbb{C}$, and $x_{n} \stackrel{\tau_{\mu}}{\rightarrow} x$ in $X$. If $\lambda_{n} \rightarrow 0$ or $\left\|x_{n}-x\right\| \rightarrow 0$ then $\lambda_{n} x_{n} \rightarrow \lambda x$ with respect to the norm-topology and thus also with respect to $\tau_{\mu}$. If $\inf \left|\lambda_{n}\right|>0, x_{n}-x \stackrel{\text { asy }}{\sim} l^{1}$, and inf $\left\|x_{n}-x\right\|>0$ then $\lambda_{n} x_{n}-\lambda_{n} x \stackrel{\text { asy }}{\sim} l^{1}$ whence $\lambda_{n} x_{n}-\lambda x \stackrel{\text { asy }}{\sim} l^{1}$ because $\left\|\lambda_{n} x-\lambda x\right\| \rightarrow 0$ and because sequences spanning $l^{1}$ asymtotically are stable by adding norm-null sequences. Hence $\lambda_{n} x_{n} \stackrel{\tau_{\mu}}{\rightarrow} \lambda x$. Up to the usual reasoning on subsequences of subsequences this proves that $\lambda_{n} \rightarrow \lambda$ and $x_{n} \stackrel{\tau_{\mu}}{\rightarrow} x$ imply $\lambda_{n} x_{n} \stackrel{\tau_{\mu}}{\rightarrow} \lambda x$.

(h) We assume to the contrary that $\left(x_{\gamma}\right)$ does not $\tau_{\mu}$-converge to 0 . Then there is a $\tau_{\mu}$-neighborhood $\mathcal{O}$ of 0 and a subnet $\left(x_{\gamma^{\prime}}\right)$ which does not meet $\mathcal{O}$ and which still $w^{*}$-converges to $x_{s}$. But by Lemma 2 there is a 
sequence $\left(x_{\gamma_{n}^{\prime}}\right)$ that spans $l^{1}$ almost isometrically i.e. $x_{\gamma_{n}^{\prime}} \stackrel{\tau_{\mu}}{\rightarrow} 0$ hence $\left(x_{\gamma_{n}^{\prime}}\right)$ does meet $\mathcal{O}$.

(i) Let $\mathcal{U}$ be a $w^{*}$-neighbourhood of $x^{\prime \prime}$. Then there exists a $x_{s} \in \mathrm{B}_{X_{s}} \cap \mathcal{U}$ and $\mathcal{U}$ is also a $w^{*}$-neighbourhood of $x_{s}$. Let $\left(x_{\gamma}\right) \subset \mathrm{B}_{X}$ be a net with $w^{*}$-limit $x_{s}$ and such that $\left\|x_{\gamma}\right\|=\left\|x_{s}\right\|$. Let $\mathcal{V}$ be a $\tau_{\mu}$-neighbourhood of 0 . Then $x_{\gamma} \stackrel{\tau_{\mu}}{\rightarrow} 0$ by (h) and there is $\gamma_{0}$ such that $x_{\gamma} \in \mathcal{U}$ and $x_{\gamma} \in \mathcal{V}$ for all $\gamma \succeq \gamma_{0}$. That is $\mathcal{V} \cap \mathcal{U} \cap \mathrm{B}_{X} \neq \emptyset$ whence the assertion.

Remarks:

1. Part (h) of the Lemma above corresponds to [13, IV.3.7], part (i) to [10, Lem. 2.2]. Since in general $\mathrm{B}_{X_{s}}$ is not $w^{*}$-closed there are $\tau_{\mu}$-null sequences which differ from the ones described in part $(\mathrm{h})$. It is tempting to suppose that the $\tau_{\mu}$-null nets are exactly those that admit $w^{*}$-limits in ${\overline{\mathrm{B}} X_{s}}^{*}$ but at the time of this writing this is not at all clear.

2. There exists a non-Hausdorff Fréchet space in which every sequence has at most one limit [8, 1.6E]. Therefore the question whether $\tau_{\mu}$ is Hausdorff is not necessarily trivial.

3. If $X, Y$ are two L-embedded Banach spaces, $Y \subset X$ a subspace of $X$, then by (b) and (e) one can identify the intrinsic abstract measure topology of $Y$ with the relative topology of the abstract measure topology of $X$. Therefore, in theorems like Theorem 13 or Proposition 16 it is no longer necessary to consider a sourrounding L-embedded Banach space like $\mathrm{L}^{1}(\mu)$ in the corresponding theorems [13, IV.3.10] or [10, Prop. 2.1]. This observation sheds also some light on the remarks after [13, IV.3.5] and after [13, Def. IV.4.2] on the use of "nicely placed" and "L-embedded".

4. It might be usefull for other purposes to modify Definition 3. For example one could replace "asymptotically" by "almost isometrically" in Definition 3; by [23, Cor. 3] this would give the same topology for L-embedded spaces. As a less trivial modification one could first restrict Definition 3 to bounded subsets of a Banach space $X$ and then define the abstract measure topology on the whole of $X$ as the inductive limit of the family $\left.\tau_{\mu}\right|_{n \mathrm{~B}_{x}}$. In this case, the results of this paper would remain valid up to some minor modifications because in all proofs except for Lemma 5 only the restriciton of $\tau_{\mu}$ to the unit ball is considered. In passing we note that we restrict our attention to bounded sets mainly because the characterization of measure-null sequences in 24] does not work for unbounded sequences, see the second remark after the proof of Theorem 1 in [24.

We end this section with a modest attempt to get closer to the sequential structure of $\tau_{\mu}$. In case the addition is $\tau_{\mu}$-continuous, at least on bounded sets $\tau_{\mu}$ gives a Fréchet space. This applies to von Neumann preduals which in $\S 6$ below will be shown to have $\tau_{\mu}$-continuous addition.

Lemma 6 Let $X$ be an L-embedded Banach space. If the addition is $\tau_{\mu}$-continuous then the restriction of $\tau_{\mu}$ to a bounded subset of $X$ makes this set a Fréchet space.

Proof: To avoid trivialities we consider a bounded sequence $x_{n} \stackrel{\text { asy }}{\sim} l^{1}$ in $X$, and a uniformely bounded sequence of sequences $\left(x_{m}^{(n)}\right)_{m \in \mathbb{N}}$ such that $x_{m}^{(n)}-x_{n} \stackrel{\text { asy }}{\sim} l^{1}$ for all $n \in \mathbb{N}$. It is enough to show the existence of a sequence $\left(m_{n}\right)$ such that $x_{m_{n}}^{(n)} \stackrel{\tau_{\mu}}{\rightarrow} 0$ because this will prove that $\left(X, \mathcal{C}, l i^{*}\right)$ of the proof of Theorem 4 satisfies (L4).

We set $y_{m}^{(n)}=x_{m}^{(n)}-x_{n}$. Since all $x_{m}^{(n)}$ are uniformely bounded there is no loss of generality if we suppose that $\left\|y_{m}^{(n)}\right\|=1$ for all $m$ and all $n$. By Lemma 2 each universal net $\left(y_{m_{\gamma}}^{(n)}\right)_{\gamma \in \Gamma} w^{*}$-converges to a limit $y_{s}^{(n)} \in X_{s}$ of norm one. What follows is a straightforward modification of the proof of [23, Th. 2]. Let $\left(\delta_{n}\right)$ be a sequence of strictly positive numbers in $] 0,1]$ converging to 0 . Set $\eta_{1}=\frac{1}{3} \delta_{1}$ and $\eta_{n+1}=\frac{1}{3} \min \left(\eta_{n}, \delta_{n+1}\right)$ for $n \in \mathbb{N}$. By induction over $n \in \mathbb{N}$ one constructs $m_{n} \in \mathbb{N}$ such that

$$
\left(\sum_{i=1}^{n}\left(1-\delta_{i}\right)\left|\alpha_{i}\right|\right)+\eta_{n} \sum_{i=1}^{n}\left|\alpha_{i}\right| \leq\left\|\sum_{i=1}^{n} \alpha_{i} y_{m_{i}}^{(i)}\right\| \text { for all } n \in \mathbb{N}, \alpha_{i} \in \mathbb{C} .
$$

The first induction step is settled by setting $m_{1}=1$. For the induction step $n \mapsto n+1$ fix an element $\alpha=\left(\alpha_{i}\right)_{i=1}^{n+1}$ in the unit sphere of $l_{n+1}^{1}$ such that $\alpha_{n+1} \neq 0$. The $w^{*}$-convergence (along $\left.\gamma\right)$ of $\left(\sum_{i=1}^{n} \alpha_{i} y_{m_{i}}^{(i)}\right)+$ $\alpha_{n+1} y_{m_{\gamma}}^{(n+1)}$ to $\left(\sum_{i=1}^{n} \alpha_{i} y_{m_{i}}^{(i)}\right)+\alpha_{n+1} y_{s}^{(n+1)}$ yields

$$
\begin{aligned}
\liminf _{\gamma}\left\|\left(\sum_{i=1}^{n} \alpha_{i} y_{m_{i}}^{(i)}\right)+\alpha_{n+1} y_{m_{\gamma}}^{(n+1)}\right\| & \geq\left\|\left(\sum_{i=1}^{n} \alpha_{i} y_{m_{i}}^{(i)}\right)+\alpha_{n+1} y_{s}^{(n+1)}\right\| \\
& =\left\|\sum_{i=1}^{n} \alpha_{i} y_{m_{i}}^{(i)}\right\|+\left|\alpha_{n+1}\right|
\end{aligned}
$$




$$
\text { 国 }\left(\sum_{i=1}^{n+1}\left(1-\delta_{i}\right)\left|\alpha_{i}\right|\right)+\min \left(\eta_{n}, \delta_{n+1}\right)
$$

whence

$$
\left\|\left(\sum_{i=1}^{n} \alpha_{i} y_{m_{i}}^{(i)}\right)+\alpha_{n+1} y_{m}^{(n+1)}\right\| \geq\left(\sum_{i=1}^{n+1}\left(1-\delta_{i}\right)\left|\alpha_{i}\right|\right)+3 \eta_{n+1}
$$

for infinitely many $m$. This extends to a finite $\eta_{n+1}$-net $\left(\alpha^{l}\right)_{l=1}^{L_{n+1}}$ in the unit sphere of $l_{n+1}^{1}$ with $2 \eta_{n+1}$ instead of $3 \eta_{n+1}$, to all $\alpha$ in the unit sphere of $l_{n+1}^{1}$ with $\eta_{n+1}$ instead of $2 \eta_{n+1}$, and finally to all $\alpha \in l_{n+1}^{1}$. The details are the same as in the proof of [23, Th. 2].

This ends the induction. By (1) we have $y_{m_{n}}^{(n)} \stackrel{\tau_{\mu}}{\rightarrow} 0$. Hence $x_{m_{n}}^{(n)}=y_{m_{n}}^{(n)}+x_{n} \stackrel{\tau_{\mu}}{\rightarrow} 0$ because addition is supposed to be $\tau_{\mu}$-continuous.

$\S 4$ Section IV.3 of 13 (partly) revisited

Proposition 7 generalizes some well known facts, see e.g. [7, Th. IV.8.12] for (a) and [13, p. 202] for (b).

Proposition 7 Let $X$ be an L-embedded Banach space with its abstract measure topology $\tau_{\mu}$. Then the following statements hold.

(a) A sequence converges in norm if (and only if) it converges both weakly and with respect to $\tau_{\mu}$, and all limits coincide.

(b) A norm closed subspace $Y \subset X$ is reflexive if and only if $\tau_{\mu}$ and the norm topology coincide on the unit ball of $Y$.

Proof: (a) The statement is almost immediate from the definiton of $\tau_{\mu}$ : First remark that a sequence which $\tau_{\mu}$-converges to 0 contains a subsequence which either converges to 0 in norm or is uniformely bounded away from 0 in norm and spans $l^{1}$ (asymptotically); but the latter case is excluded if the sequence also converges weakly (to whatever limit) because $l^{1}$-bases do not converge weakly. Now let $\left(x_{n}\right)$ be a sequence in $X$, let $x, y \in X$ be such that both $x_{n} \stackrel{\tau_{\mu}}{\rightarrow} x$ and $x_{n} \stackrel{w}{\rightarrow} y$. Then by what has just been remarked, for each subsequence $\left(x_{n_{k}}\right)$ there is a subsequence $\left(x_{n_{k_{l}}}\right)$ such that $x_{n_{k_{l}}}-x \rightarrow 0$ in norm for any subsequence $\left(x_{n_{k}}\right)$ whence $x=y$ and the assertion follows.

(b) By $\tau_{\|\cdot\|}$ we denote the norm topology on $X$. Let $Y$ be reflexive. To show that $\tau_{\mu}$ and $\tau_{\|\cdot\|}$ coincide on the unit ball $\mathrm{B}_{Y}$ of $Y$ it is enough to show that each subsequence of a $\tau_{\mu}$-convergent sequence in $\mathrm{B}_{Y}$ admits a subsequence which converges in norm to the same limit. But if $Y$ is reflexive then each bounded sequence contains a weakly convergent subsequence which if the sequence is also $\tau_{\mu}$-convergent converges in norm by (a). Thus $\tau_{\mu}$ and $\tau_{\|\cdot\|}$ coincide on $\mathrm{B}_{Y}$.

Conversely suppose that $\tau_{\mu}$ and $\tau_{\|\cdot\|}$ coincide on $\mathrm{B}_{Y}$. In order to prove that $Y$ is reflexive it is enough to prove that $Y$ does not contain isomorphic copies of $l^{1}$ because by Rosenthal's theorem [4. $\mathrm{Ch}$. XI] in the absence of $l^{1}$ each bounded sequence contains a weak Cauchy subsequence which then converges weakly by the weak sequential completeness of $X$. But if $Y$ contained an isomorphic copy of $l^{1}$ then by James' distortion theorem it would contain also an almost isometric copy of $l^{1}$. By [23, Cor. 3] $Y$ would contain an asymptotic copy of $l^{1}$. It's canonical normalized basis would $\tau_{\mu}$-converge to 0 ; finally, since $\tau_{\mu}$ and $\tau_{\|\cdot\|}$ coincide on $\mathrm{B}_{Y}$ this basis would even converge in norm to 0 , a contradiction which proves that $Y$ is reflexive.

Lemma 8 is the technical key for the other results of this section. It corresponds to [13, IV.3.1].

Lemma 8 Let $X$ be an $L$-embedded Banach space with L-projection $P$ from $X^{\prime \prime}$ onto $X$. Then for every net $\left(x_{\gamma}\right)_{\gamma \in \Gamma}$ in $X w^{*}$-converging to $x^{\prime \prime} \in X^{\prime \prime} \backslash X$ there is a bounded sequence $\left(y_{n}\right)$ in co $\left\{x_{\gamma} \mid \gamma \in \Gamma\right\}$ such that the sequence $\left(y_{n}-P x^{\prime \prime}\right)$ spans $l^{1}$ asymtotically isometrically.

Proof: Set $x=P x^{\prime \prime}, x_{s}=x^{\prime \prime}-P x^{\prime \prime}$. Choose a net $\left(z_{\gamma}\right)_{\gamma \in \Gamma}$ in $X$ such that $z_{\gamma} \stackrel{w^{*}}{\rightarrow} x_{s}$ and $\left\|z_{\gamma}\right\|=\left\|x_{s}\right\|$. We assume without loss of generality that both nets $\left(x_{\gamma}\right),\left(z_{\gamma}\right)$ are indexed by the same directed set $\Gamma$. Then $x_{\gamma}-z_{\gamma} \stackrel{w}{\rightarrow} x$.

The idea of the proof is that on one hand by the theorem of Hahn-Banach the net $\left(x_{\gamma}-z_{\gamma}\right)$ admits convex combinations which converge to $x$ in norm and that on the other hand by a slight modification of Godefroy's construction the corresponding convex combinations of the $x_{\gamma}$ can be chosen so to span $l^{1}$ asymtotically. 
Here are the details.

Since $x^{\prime \prime} \notin X$ we have $x_{s} \neq 0$ and thus without loss of generality we suppose $\left\|x_{s}\right\|=\left\|z_{\gamma}\right\|=1$. Let $\left(\delta_{n}\right)$ be a sequence of numbers in ]0,1[ convergent to 0 . Set $\eta_{1}=\frac{1}{4} \delta_{1}$ and $\eta_{n+1}=\frac{1}{4} \min \left(\eta_{n}, \delta_{n+1}\right)$ for $n \in \mathbb{N}$. By induction over $n \in \mathbb{N}$ we will construct finite sets $A_{n} \subset \mathbb{N}$, finite sequences $\left(\lambda_{k}\right)_{k \in A_{n}}$ in $[0,1]$ and $\left(\gamma_{k}\right)_{k \in A_{n}}$ in $\Gamma$ such that

$$
\begin{aligned}
\sum_{k \in A_{n}} \lambda_{k}= & 1, G_{i} \cap G_{n}=\emptyset, G_{n} \subset \Gamma_{n} \quad \forall i<n \\
& \left\|\left(y_{n}-x\right)-\sum_{k \in A_{n}} \lambda_{k} z_{\gamma_{k}}\right\|<\eta_{n} . \\
\sum_{i=1}^{n}\left|\alpha_{i}\right|\left(1+\eta_{i}\right) \geq & \left\|\sum_{i=1}^{n} \alpha_{i}\left(y_{i}-x\right)\right\| \geq\left(\sum_{i=1}^{n}\left(1-\delta_{i}\right)\left|\alpha_{i}\right|\right)+\eta_{n} \sum_{i=1}^{n}\left|\alpha_{i}\right| \forall \alpha_{i} \in \mathbb{C}
\end{aligned}
$$

where

$$
y_{n}=\sum_{k \in A_{n}} \lambda_{k} x_{\gamma_{k}}, \quad G_{n}=\left\{\gamma_{k} \mid k \in A_{n}\right\}, \quad \Gamma_{n}=\Gamma \backslash \bigcup_{i=1}^{n-1} G_{i} \quad(=\Gamma \text { if } n=1) .
$$

For the first induction step $n=1$ we choose $x^{\prime} \in \mathrm{B}_{X^{\prime}}$ such that $1=\left\|x_{s}\right\| \geq \operatorname{Re} x_{s}\left(x^{\prime}\right)>1-\delta_{1}+2 \eta_{1}$. The $w^{*}$-convergence of $\left(z_{\gamma}\right)$ to $x_{s}$ yields $\beta_{1} \in \Gamma$ such that

$$
\operatorname{Re} x^{\prime}\left(z_{\gamma}\right)>1-\delta_{1}+2 \eta_{1} \forall \gamma \succeq \beta_{1}
$$

The net $\left(\left(x_{\gamma}-x\right)-z_{\gamma}\right)_{\gamma \succeq \beta_{1}} w$-converges to 0 thus by the theorem of Hahn-Banach we find a convex combination $y_{1}=\sum_{k \in A_{1}} \lambda_{k} x_{\gamma_{k}}$ such that

$$
\left\|\left(y_{1}-x\right)-\sum_{k \in A_{1}} \lambda_{k} z_{\gamma_{k}}\right\|<\eta_{1}
$$

Thus (4, $n=1$ ) follows from $\left\|y_{1}-x\right\| \leq\left\|\sum_{k \in A_{1}} \lambda_{k} z_{\gamma_{k}}\right\|+\eta_{1} \leq 1+\eta_{1}$ and from

$$
\begin{aligned}
\left\|y_{1}-x\right\| & >\left\|\sum_{k \in A_{1}} \lambda_{k} z_{\gamma_{k}}\right\|-\eta_{1} \\
& \geq-\eta_{1}+\operatorname{Re} \sum_{k \in A_{1}} \lambda_{k} x^{\prime}\left(z_{\gamma_{k}}\right) \\
& >-\eta_{1}+\sum_{k \in A_{1}} \lambda_{k}\left(1-\delta_{1}+2 \eta_{1}\right)=1-\delta_{1}+\eta_{1} .
\end{aligned}
$$

For the induction step $n \mapsto n+1$ we suppose $A_{i} \subset \Gamma,\left(\lambda_{k}\right)_{k \in A_{i}} \subset[0,1], G_{i} \subset \Gamma$ to be constructed according to (2) - (4) for $i=1, \ldots, n$.

First we consider the $w$-convergent net $\left(x_{\gamma}-z_{\gamma}\right)_{\gamma \in \Gamma_{n+1}}$. By the theorem of Hahn-Banach we can choose a finite set $A_{n+1} \subset \mathbb{N}$, numbers $\left(\lambda_{k}\right)_{k \in A_{n+1}} \subset[0,1]$ and indices $\left(\gamma_{k}\right)_{k \in A_{n+1}} \subset \Gamma_{n+1}$ such that (2) and (3) hold for $n+1$. Together with $(4, n)$ this gives the first inequality of $(4, n+1)$.

We fix an element $\alpha=\left(\alpha_{i}\right)$ in the unit sphere of $l_{n+1}^{1}$ such that $\alpha_{n+1} \neq 0$ and use the L-decomposition of $X^{\prime \prime}=X \oplus_{1} X_{s}$ in order to get

$$
\begin{aligned}
\left\|\left(\sum_{i=1}^{n} \alpha_{i}\left(y_{i}-x\right)\right)+\alpha_{n+1} x_{s}\right\| & =\left\|\sum_{i=1}^{n} \alpha_{i}\left(y_{i}-x\right)\right\|+\left\|\alpha_{n+1} x_{s}\right\| \\
& \underbrace{(1)}_{i=1}\left(\sum_{i=1}^{n}\left(1-\delta_{i}\right)\left|\alpha_{i}\right|\right)+\eta_{n}\left(\sum_{i=1}^{n}\left|\alpha_{i}\right|\right)+\left|\alpha_{n+1}\right| \\
& =\left(\sum_{i=1}^{n+1}\left(1-\delta_{i}\right)\left|\alpha_{i}\right|\right)+\eta_{n}-\left(\eta_{n}-\delta_{n+1}\right)\left|\alpha_{n+1}\right| \\
& \geq\left(\sum_{i=1}^{n+1}\left(1-\delta_{i}\right)\left|\alpha_{i}\right|\right)+\min \left(\eta_{n}, \delta_{n+1}\right) \\
& =\left(\sum_{i=1}^{n+1}\left(1-\delta_{i}\right)\left|\alpha_{i}\right|\right)+4 \eta_{n+1}
\end{aligned}
$$


because $\|\alpha\|=1$ and $\left|\alpha_{n+1}\right| \leq 1$. Thus there is $x^{\prime} \in \mathrm{B}_{X^{\prime}}$ (depending on $\alpha$ ) such that

$$
\begin{aligned}
\left\|\left(\sum_{i=1}^{n} \alpha_{i}\left(y_{i}-x\right)\right)+\alpha_{n+1} x_{s}\right\| & \geq \operatorname{Re}\left(\left(\sum_{i=1}^{n} \alpha_{i}\left(y_{i}-x\right)\right)+\alpha_{n+1} x_{s}\right)\left(x^{\prime}\right) \\
& >\left(\sum_{i=1}^{n+1}\left(1-\delta_{i}\right)\left|\alpha_{i}\right|\right)+3 \eta_{n+1} .
\end{aligned}
$$

Then the $w^{*}$-convergence (along $\left.\gamma \in \Gamma_{n+1}\right)$ of $\left(\left(\sum_{i=1}^{n} \alpha_{i}\left(y_{i}-x\right)\right)+\alpha_{n+1} z_{\gamma}\right)$ to $\left(\sum_{i=1}^{n} \alpha_{i}\left(y_{i}-x\right)\right)+\alpha_{n+1} x_{s}$ yields $\beta \in \Gamma_{n+1}$ (depending on $\alpha$ and $x^{\prime}$ ) such that

$$
\operatorname{Re}\left(x^{\prime}\left(\left(\sum_{i=1}^{n} \alpha_{i}\left(y_{i}-x\right)\right)+\alpha_{n+1} z_{\gamma}\right)\right)>\left(\sum_{i=1}^{n+1}\left(1-\delta_{i}\right)\left|\alpha_{i}\right|\right)+3 \eta_{n+1} \quad \forall \gamma \succeq \beta .
$$

Choose a finite $\eta_{n+1}$-net $\left(\alpha^{l}\right)_{l=1}^{L_{n+1}}$ in the unit sphere of $l_{n+1}^{1}$ in the sense that for each $\alpha$ in the unit sphere of $l_{n+1}^{1}$ there is $l \leq L_{n+1}$ such that $\left\|\alpha-\alpha^{l}\right\|=\sum_{i=1}^{n+1}\left|\alpha_{i}-\alpha_{i}^{l}\right|<\eta_{n+1}$. Then we may repeat the reasoning above finitely many times for $l=1, \ldots, L_{n+1}$ in order to get $\beta_{n+1} \in \Gamma_{n+1}$ and $x_{l}^{\prime} \in \mathrm{B}_{X^{\prime}}$ for $l \leq L_{n+1}$ such that

$$
\operatorname{Re}\left(x_{l}^{\prime}\left(\left(\sum_{i=1}^{n} \alpha_{i}^{l}\left(y_{i}-x\right)\right)+\alpha_{n+1}^{l} z_{\gamma}\right)\right)>\left(\sum_{i=1}^{n+1}\left(1-\delta_{i}\right)\left|\alpha_{i}^{l}\right|\right)+3 \eta_{n+1} \quad \forall l \leq L_{n+1}, \quad \gamma \succeq \beta_{n+1} .
$$

For each $l \leq L_{n+1}$ we get that

$$
\begin{aligned}
\left\|\sum_{i=1}^{n+1} \alpha_{i}^{l}\left(y_{i}-x\right)\right\| & \stackrel{(3)}{\geq}-\eta_{n+1}+\left\|\left(\sum_{i=1}^{n} \alpha_{i}^{l}\left(y_{i}-x\right)\right)+\alpha_{n+1}^{l} \sum_{k \in A_{n+1}} \lambda_{k} z_{\gamma_{k}}\right\| \\
& =-\eta_{n+1}+\left\|\sum_{k \in A_{n+1}} \lambda_{k}\left(\left(\sum_{i=1}^{n} \alpha_{i}^{l}\left(y_{i}-x\right)\right)+\alpha_{n+1}^{l} z_{\gamma_{k}}\right)\right\| \\
& \geq-\eta_{n+1}+\sum_{k \in A_{n+1}} \lambda_{k} \operatorname{Re} x_{l}^{\prime}\left(\left(\sum_{i=1}^{n} \alpha_{i}^{l}\left(y_{i}-x\right)\right)+\alpha_{n+1}^{l} z_{\gamma_{k}}\right) \\
& \text { (6) }\left(\sum_{i=1}^{n+1}\left(1-\delta_{i}\right)\left|\alpha_{i}^{l}\right|\right)+2 \eta_{n+1}
\end{aligned}
$$

For an arbitrary $\alpha$ in the unit sphere of $l_{n+1}^{1}$ choose $l \leq L_{n+1}$ such that $\left\|\alpha-\alpha^{l}\right\|<\eta_{n+1}$. Then

$$
\begin{aligned}
\left\|\sum_{i=1}^{n+1} \alpha_{i}\left(y_{i}-x\right)\right\| & \geq\left\|\sum_{i=1}^{n+1} \alpha_{i}^{l}\left(y_{i}-x\right)\right\|-\left\|\sum_{i=1}^{n+1}\left(\alpha_{i}-\alpha_{i}^{l}\right)\left(y_{i}-x\right)\right\| \\
& \geq\left(\sum_{i=1}^{n+1}\left(1-\delta_{i}\right)\left|\alpha_{i}\right|\right)+2 \eta_{n+1}-\left\|\alpha-\alpha^{l}\right\| \\
& \geq\left(\sum_{i=1}^{n+1}\left(1-\delta_{i}\right)\left|\alpha_{i}\right|\right)+\eta_{n+1} \\
& =\left(\sum_{i=1}^{n+1}\left(1-\delta_{i}\right)\left|\alpha_{i}\right|\right)+\eta_{n+1} \sum_{i=1}^{n+1}\left|\alpha_{i}\right| .
\end{aligned}
$$

This extends to all scalars $\alpha_{i} \in \mathbb{C}$ and thus ends the induction. The sequence $\left(y_{n}\right)$ is bounded because of (3). By (4) the sequence $\left(y_{n}-x\right)$ is easily seen to span $l^{1}$ asymptotically. This ends the proof.

Remark: The proof yields not only $y \in \operatorname{co}\left\{x_{\gamma} \mid \gamma \in \Gamma\right\}$ but separated blocks $y_{n}=\sum_{k \in A_{n}} \lambda_{k} x_{\gamma_{k}}$ where the sets $\left\{x_{\gamma_{k}} \mid k \in A_{n}\right\}$ are pairwise disjoint. Moreover one can obtain, given a sequence $\left(\gamma_{n}^{\prime}\right)$ in $\Gamma$, that $x_{\gamma_{k}} \succeq \gamma_{n}^{\prime}$ for $k \in A_{n}$.

In general the unit ball of $X$ is not $\tau_{\mu}$-compact; the Rademacher functions $r_{n}$ in $\mathrm{L}^{1}([0,1])$ which are bounded without having a measure convergent subsequence provide a counterexample. [If $\left(r_{n}\right)$ contained a measure convergent subsequence this subsequence would admit a norm convergent subsequence by Proposition 7 (a) because $\left(r_{n}\right)$ spans $l^{2}$ isomorphically.] But we have: 
Corollary 9 Every bounded sequence in an L-embedded Banach space admits a sequence of convex combinations which converges with respect to the measure topology.

Proof: Let $\left(x_{n}\right)$ be a bounded sequence in an L-embedded space $X$ and let $\left(x_{n_{\gamma}}\right)$ be a universal net that $w^{*}$-converges to $x^{\prime \prime} \in X^{\prime \prime}$ by the $w^{*}$-compactness of $\mathrm{B}_{X^{\prime \prime}}$. If $x^{\prime \prime} \in X$ then this net $w$-converges, admits norm-convergent convex combinations and we are done in this case. Otherwise $x^{\prime \prime}$ lies in $X^{\prime \prime} \backslash X$ and one applies Lemma 8 to get a sequence $\left(y_{n}\right)$ in co $\left\{x_{n} \mid n \in \mathbb{N}\right\}$ which $\tau_{\mu}$-converges to $P x^{\prime \prime}$.

Corollary 9 corresponds to [13, p. 202]. In this context there is a natural

Question: Does Komlos' theorem hold accordingly? More precisely, given a bounded sequence in an Lembedded space, does it admit a aubsequence whose Cesaro (=arithmetic) means converge with respect to the measure topology?

Note that by (a) of Proposition 0 Komlos' theorem implies the weak Banach-Saks property (which by definition claims that a $w$-convergent sequence admits a subsequence whose Cesaro means converge in norm, see for example [1], 过, p. 112, 121], [2]). By Rosenthal's $l^{1}$-theorem the weak Banach-Saks property is also half a converse to Komlos' theorem, that is by Rosenthal's $l^{1}$-theorem a bounded sequence in an L-embedded space admits a subsequence which is either equivalent to the standard basis of $l^{1}$ or converges weakly; but if one supposes the weak Banach-Saks property to hold then in the second case of a $w$-convergent sequence there are Cesaro means that converge in norm whence with respect to the measure topology.

There is another related

Question: Does the Kadec-Pełczyński subsequence decomposition (sometimes also called the Kadec-Pełczyński splitting lemma) hold accordingly? This lemma says that a bounded sequence $\left(f_{n}\right)$ in $\mathrm{L}^{1}([0,1])$ admits a subsequence $\left(f_{n_{k}}\right)$ which can be decomposed in the following sense: there are two bounded sequences $\left(g_{k}\right)$, $\left(h_{k}\right)$ in $\mathrm{L}^{1}([0,1])$ such that $f_{n_{k}}=g_{k}+h_{k}$, the $g_{k}$ are pairwise disjoint, and $\left(h_{k}\right)$ converges weakly. In a recent preprint Randrianantoanina [26] showed the Kadec-Pełczyński subsequence decomposition for preduals of von Neumann algebras. Since the latter are known to have the weak Banach-Saks property [2], Komlos' theorem follows almost immediately from Randrianantoanina's result for von Neumann preduals, see Proposition 24 below.

The following theorem generalizes a theorem of Buhvalov-Lozanovskii ([3], [13, IV.3.4]). As in [13, IV.3.4] the implication (ii) $\Rightarrow$ (i) holds also for unbounded $C$.

Theorem 10 Let $X$ be an L-embedded Banach space with L-projection $P$ (on $X^{\prime \prime}$ with range $X$ ) and endowed with its abstract measure topology $\tau_{\mu}$. For a norm closed bounded convex set $C \subset X$ the following two assertions are equivalent.

(i) $P \bar{C}^{w^{*}}=C$ where $w^{*}$ refers to the $w^{*}$-topology of $X^{\prime \prime}$.

(ii) $C$ is $\tau_{\mu}$-closed.

Proof: (i) $\Rightarrow$ (ii) Take $c_{n} \in C, x \in X$ with $c_{n} \stackrel{\tau_{\mu}}{\rightarrow} x$. It is enough to show that $x \in C$ because closedness and sequential closedness coincide for $\tau_{\mu}$. If the $\tau_{\mu}$ - null sequence $\left(c_{n}-x\right)$ contains a norm convergent subsequence then we are done because in the norm topology $C$ is closed. Otherwise an appropriate subsequence $\left(c_{n_{k}}-x\right)$ spans $l^{1}$ asymptotically and $\inf \left\|c_{n_{k}}-x\right\|>0$. By $w^{*}$-compactness of $\bar{C}^{w^{*}}$ there is a net $\left(c_{n_{k_{\gamma}}}-x\right)_{\gamma \in \Gamma}$ on $\mathcal{S}=\left\{c_{n_{k}}-x \mid k \in \mathbb{N}\right\}$ that $w^{*}$-converges to $\bar{c}-x \in \bar{C}^{w^{*}}-x$. By Lemma 2 we have that $\bar{c}-x \in X_{s}$ because $C$ is bounded. Thus $P(\bar{c}-x)=0$ and $x=P x=P \bar{c} \in C$ by hypothesis.

(ii) $\Rightarrow$ (i) This implication is essentially Lemma 8: If $\left(c_{\gamma}\right)_{\gamma \in \Gamma}$ is a $w^{*}$-convergent net in $C$ with limit $\bar{c}$ it is enough to prove that $P \bar{c} \in C$ because the inclusion $C \subset P \bar{C}^{w^{*}}$ is trivial. If $\bar{c} \in X$ then there is a sequence of convex combinations of the $c_{\gamma}$ convergng to $\bar{c}$ in norm whence $\bar{c} \in C$. Otherwise, if $\bar{c} \in X^{\prime \prime} \backslash X$, by Lemma 8 there is a sequence $\left(d_{n}\right) \in \operatorname{co}\left\{c_{\gamma} \mid \gamma \in \Gamma\right\} \subset C$ which $\tau_{\mu}$-converges to $P \bar{c}$ hence $P \bar{c} \in C$ because $C$ is $\tau_{\mu}$-closed.

Corollary 11 corresponds to [13, IV.3.5]) for the case $X=\mathrm{L}^{1}(\Omega, \Sigma, \mu)$.

Corollary 11 Let $X$ be an L-embedded Banach space endowed with its abstract measure topology $\tau_{\mu}$. Then a norm closed subspace $Y \subset X$ is L-embedded if and only if its unit ball $\mathrm{B}_{Y}$ is $\tau_{\mu}$-closed.

The proof is immediate from Theorem 10 with $C=\mathrm{B}_{Y}$ and from Li's criterion Lemma \&.

Let $X$ be a Banach space admitting an abstract measure topology $\tau_{\mu}$. Then we define

$$
X^{\#}=\left\{x^{\prime} \in X^{\prime}\left|x^{\prime}\right|_{\mathrm{B}_{X}} \text { is } \tau_{\mu} \text { continuous }\right\} .
$$


Remarks:

1. $X^{\#}$ is a closed subspace of $X^{\prime}$. (The proof is left to the reader.)

2. If $X$ is a subspace of an L-embedded Banach space then one has $X^{\#}=X^{\prime}$ if and only if $X$ does not contain copies of $l^{1}$. For, in the absence of $l^{1}, \tau_{\mu}$ coincides with the norm topology hence $X^{\#}=X^{\prime}$. Conversely, if $X$ contains a copy of $l^{1}$ then by James' destortion theorem it contains also an almost isometric copy $U$ of $l^{1}$ spanned by a normalized basis $\left(u_{n}\right)$. Since $X$ is contained in an L-embedded space, by [23, Cor. 3] we (may pass to an appropriate subsequence and) suppose that $u_{n} \stackrel{\tau_{\mu}}{\rightarrow} 0$. Let $x^{\prime} \in X^{\prime}$ be a Hahn-Banach extension of the functional on $U$ which corresponds to $1 \in l^{\infty}$. Then $x^{\prime}$ is not $\tau_{\mu}$-continuous on $\mathrm{B}_{X}$ since $u_{n} \stackrel{\tau_{\mu}}{\rightarrow} 0$ but $x^{\prime}\left(u_{n}\right) \rightarrow 1$. (Compare also with [13, Rem. (b) p. 186].)

Proposition 12 Let $X$ be an L-embedded Banach space (with L-decomposition $X^{\prime \prime}=X \oplus_{1} X_{s}$ ) endowed with its abstract measure topology $\tau_{\mu}$. Then

$$
X^{\#}=\left(X_{s}\right)_{\perp}\left(\subset X^{\prime}\right) .
$$

Proof: " $\subset$ "Take $x^{\prime} \in X^{\#}$ and $x_{s} \in X_{s}$. To prove the inclusion we show that $x_{s}\left(x^{\prime}\right)=0$.

Let $\left(x_{\gamma}\right)$ be a net that $w^{*}$-converges to $x_{s}$ with $\left\|x_{\gamma}\right\|=\left\|x_{s}\right\|$. But then, by Lemma 2 there is a sequence $\left(x_{\gamma_{n}}\right)$ that spans $l^{1}$ asymptotically. Hence $x_{\gamma_{n}} \stackrel{\tau_{\mu}}{\rightarrow} 0$ and $x^{\prime}\left(x_{\gamma_{n}}\right) \rightarrow 0$ since $x^{\prime}$ is $\tau_{\mu}$-continuous on bounded sets. This proves that $x^{\prime \prime}\left(x^{\prime}\right)=0$. (In passing we note that $x_{\gamma} \stackrel{\tau_{\mu}}{\rightarrow} 0$ by (h) of Lemma 5 but that one can not infer from this that $x^{\prime \prime}\left(x^{\prime}\right)=0$ because it is not clear whether a $\tau_{\mu}$-convergent net has a unique limit.) " $\supset$ " Assume that there is $\left.x^{\prime} \in\left(X_{s}\right)\right)_{\perp}$ that is not $\tau_{\mu}$-continuous on $\mathrm{B}_{X}$. Then by the definiton of $\tau_{\mu}$ there are $\varepsilon>0$ and a sequence $\left(x_{n}\right)$ in $\mathrm{B}_{X}$ such that $x_{n} \stackrel{\tau_{\mu}}{\rightarrow} 0$ but $\left|x^{\prime}\left(x_{n}\right)\right|>\varepsilon$ for all $n \in \mathbb{N}$. Still by definiton of $\tau_{\mu}$ and because $x^{\prime}$ is norm-continuous we suppose that $\left(x_{n}\right)$ spans $l^{1}$ almost isometrically. By $w^{*}$-compactness of $\mathrm{B}_{X^{\prime \prime}}$ there exists a $w^{*}$-accumulation point $x^{\prime \prime} \in \mathrm{B}_{X^{\prime \prime}}$ of $\left\{x_{n} \mid n \in \mathbb{N}\right\}$. Let $\left(x_{n_{\gamma}}\right)$ be a net $w^{*}$-converging to $x^{\prime \prime}$. By Lemma 2, $x^{\prime \prime} \in X_{s}$. Thus $x^{\prime}\left(x_{n_{\gamma}}\right) \rightarrow x^{\prime \prime}\left(x^{\prime}\right)=0$ by hypothesis. This contradicts $\left|x^{\prime}\left(x_{n}\right)\right|>\varepsilon$ and proves that $x^{\prime}$ is $\tau_{\mu}$-continuous on $\mathrm{B}_{X}$.

Theorem 13 (see also [13, IV.3.10]) was proved in [11] for nicely placed (=L-embedded) subspaces $X$ of $\mathrm{L}^{1}(\Omega, \Sigma, \mu), \mu$ finite. For its proof Proposition 12 plays the same rôle as [13, IV.3.9] in the proof of [13, IV.3.10]. Recall that if an L-embedded space admits a predual then this predual need not be M-embedded ([13, p. 102]).

Theorem 13 Let $X$ be an L-embedded Banach space endowed with its abstract measure topology $\tau_{\mu}$. The following assertions are equivalent.

(i) $X$ is (isometrically isomorphic to) the dual of an M-embedded Banach space $Z$.

(ii) $X^{\#}$ separates $X$.

If (i) and (ii) are satisfied $Z$ is (isometrically isomorphic to) $X^{\#}$.

Proof: (i) $\Rightarrow\left(\right.$ ii): By [13, III.1.3] the L-projections on $X^{\prime \prime}$ and on $Z^{\prime \prime \prime}$ with kernel $X_{s}=Z^{\perp} \subset X^{\prime \prime}$ can be identified. Thus $Z=\left(Z^{\perp}\right)_{\perp}=\left(X_{s}\right)_{\perp}=X^{\#}$ by Proposition 12 in particular, $X^{\#}$ separates $X$.

(ii) $\Rightarrow$ (i): By [13, IV.1.9] it is enough to show that $X_{s}$ is $w^{*}$-closed in $X^{\prime \prime}$; then an M-embedded predual of $X$ exists and is isometrically isomorphic to $\left(X_{s}\right)_{\perp}$ whence to $X^{\#}$ by Proposition 12 . To see that $X_{s}$ is $w^{*}$-closed we take an element

$$
\bar{x}=x+x_{s} \in{\overline{X_{s}}}^{w^{*}}=\left(\left(X_{s}\right)_{\perp}\right)^{\perp}=\left(X^{\#}\right)^{\perp}
$$

with $x \in X, x_{s} \in X_{s}$. Then $x=\bar{x}-x_{s} \in\left(X^{\#}\right)^{\perp} \cap X=\left(X^{\#}\right)_{\perp}=\{0\}$ where the latter equality comes from the fact that $X^{\#}$ separates $X$. Thus $\bar{x}=x_{s} \in X_{s}$ which proves that $X_{s}$ is $w^{*}$-closed in $X^{\prime \prime}$.

Analogously to the $l^{1}$-case we say that a sequence $\left(x_{n}\right)$ of nonzero elements in a Banach space $X$ spans $c_{0}$ almost (respectivley asymptotically) isometrically if there exists a sequence $\left(\delta_{m}\right)$ in $[0,1$ [ tending to 0 such that $\left(1-\delta_{m}\right) \max _{m \leq n \leq m^{\prime}}\left|\alpha_{n}\right| \leq\left\|\sum_{n=m}^{m^{\prime}} \alpha_{n} \frac{x_{n}}{\left\|x_{n}\right\|}\right\| \leq\left(1+\delta_{m}\right) \max _{m \leq n \leq m^{\prime}}\left|\alpha_{n}\right|$ for all $m \leq m^{\prime}$ (respectively such that $\max _{n \leq m}\left(1-\delta_{n}\right)\left|\alpha_{n}\right| \leq\left\|\sum_{n=1}^{m} \alpha_{n} \frac{x_{n}}{\left\|x_{n}\right\|}\right\| \leq \max _{n \leq m}\left(1+\delta_{n}\right)\left|\alpha_{n}\right|$ for all $\left.m \in \mathbb{N}\right)$.

It follows from the proof of [6, Th. 2] that the dual of an asymptotic $c_{0}$-copy is an asymptotic $l^{1}$-copy. A similar argument shows that this remains true if "asymptotic" is replaced by "almost isometric". Analogously we get 
Lemma 14 Let $X=\left[x_{n}\right]$ be an almost isometric copy of $l^{1}$ spanned by the normalized basis $\left(x_{n}\right)$. Let $x_{n}^{\prime} \in X^{\prime}$ be the biorthogonal functionals (that is $x_{n}^{\prime}\left(x_{k}\right)=\delta_{n, k}$ ). Then $\left(x_{n}^{\prime}\right)$ spans $c_{0}$ almost isometrically.

The same holds with "almost" replaced by "asymptotically".

Proof: First we deal with the case where $x_{n} \stackrel{\text { alm }}{\sim} l^{1}$.

By hypothesis there is a null sequence $\left(\delta_{m}\right) \subset[0,1[$ sucht that

$$
\left(1-\delta_{m}\right) \sum_{m}^{\infty}\left|\beta_{n}\right| \leq\left\|\sum_{m}^{\infty} \beta_{n} x_{n}\right\| \leq \sum_{m}^{\infty}\left|\beta_{n}\right|
$$

for all scalars $\beta_{n}$. Let $m \leq m^{\prime}$ be arbitrary in $\mathbb{N}$. Since $\left\|x_{n}\right\|=1$ we have the first inequality of

$$
\max _{m \leq n \leq m^{\prime}}\left|\alpha_{n}\right| \leq\left\|\sum_{n=m}^{m^{\prime}} \alpha_{n} x_{n}^{\prime}\right\| \leq\left(1-\delta_{m}\right)^{-1} \max _{m \leq n \leq m^{\prime}}\left|\alpha_{n}\right|
$$

for all scalars $\alpha_{n}$. For the second inequality we take any $x \in \mathrm{B}_{X}$ of the form $x=\sum \beta_{n} x_{n}$; then

$$
\begin{aligned}
\left|\sum_{n=m}^{m^{\prime}} \alpha_{n} x_{n}^{\prime}(x)\right| & =\left|\sum_{n=m}^{m^{\prime}} \alpha_{n} \beta_{n}\right| \leq \max _{m \leq n \leq m^{\prime}}\left|\alpha_{n}\right| \sum_{n=m}^{m^{\prime}}\left|\beta_{n}\right| \\
& \leq\left(1-\delta_{m}\right)^{-1} \max _{m \leq n \leq m^{\prime}}\left|\alpha_{n}\right|
\end{aligned}
$$

whence the second inequality of (11). It follows from (11) that $\left(x_{n}^{\prime}\right)$ spans $c_{0}$ almost isometrically.

The case in which $x_{n} \stackrel{\text { asy }}{\sim} l^{1}$ is proved similarly.

Proposition 15 An L-embedded almost isometric copy of $l^{1}$ is the dual of an $M$-embedded space which is almost isometric to $c_{0}$.

The statement remains true when "almost" is replaced by "asymptotically".

Proof: Let $X$ be an L-embedded almost isometric $l^{1}$-copy with a normalized canonical basis $\left(x_{n}\right)$. Let $\left(x_{n}^{\prime}\right)$ be the biorthogonal functionals of $\left(x_{n}\right)$ that is $x_{n}^{\prime}\left(x_{m}\right)=\delta_{n, m}$ for $n, m \in \mathbb{N}$.

Sublemma $x_{n}^{\prime} \in X^{\#}$ for all $n \in \mathbb{N}$.

Proof of the Sublemma: Suppose there is $n_{0} \in \mathbb{N}$ such that $x^{\prime}=x_{n_{0}} \notin X^{\#}$. Then there is a sequence $\left(y_{n}\right) \subset \mathrm{B}_{X}$ and there is $\varepsilon>0$ such that

$$
\left\|y_{n}\right\|=1, \quad y_{n} \stackrel{\text { asy }}{\sim} l^{1}, \quad\left|x^{\prime}\left(y_{n}\right)\right|>\varepsilon \text { for all } n \in \mathbb{N} .
$$

By [23, Lem. 1] $Y=\left[y_{n}\right]$ is L-embedded and $Y^{\perp \perp}=Y \oplus_{1} Y_{s}$ with $Y_{s}=Y^{\perp \perp} \cap X_{s}$. Let $\delta>0$ be arbitrary for the moment. Set $X_{0}=\left[x_{n}\right]_{n \geq m_{0}}$ where $m_{0}$ is such that $\left(x_{n}\right)_{n \geq m_{0}} \stackrel{1-\delta}{\sim} l^{1} . X_{0}$ is L-embedded by [23, Lem. 1] and we write $X_{0}^{\perp \perp}=X_{0} \oplus_{1}\left(X_{0}\right)_{s}$ with $\left(X_{0}\right)_{s}=X_{0}^{\perp \perp} \cap X_{s}$. Let $y_{s} \in X^{\prime \prime}$ be a $w^{*}$-accumulation point of $\left\{y_{n} \mid n \in \mathbb{N}\right\}$. By Lemma 2 we have $\left\|y_{s}\right\|=1$ and $y_{s} \in Y_{s}$. We have $y_{s} \in\left(X_{0}\right)_{s}$ because $X_{0}$ is co-finite-dimensional in $X$; furthermore, $\left|y_{s}\left(x^{\prime}\right)\right| \geq \varepsilon$. Let $\left(z_{\gamma}\right) \subset \mathrm{B}_{X_{0}}$ be a normalized net $w^{*}$-converging to $y_{s}$. After passing to an appropriate subnet we suppose that $\left|\left(y_{s}-z_{\gamma}\right)\left(x^{\prime}\right)\right|<\varepsilon / 2$ for all $\gamma$. By Lemma 2 one can extract a sequence $\left(z_{\gamma_{n}}\right)$ that spans $l^{1}$ asymptotically. Then $\left|x^{\prime}\left(z_{\gamma_{n}}\right)\right| \geq \varepsilon / 2$ for all $n$. We define an isomorphism $T: X_{0} \rightarrow\left[e_{n}\right]_{n \geq m_{0}}$ by $x_{n} \mapsto e_{n}$ where $\left(e_{n}\right)$ is the standard basis of $l^{1}$. Then $\|T\| \leq(1-\delta)^{-1}$ and $\left\|T^{-1}\right\| \leq 1$. There is $m_{1} \in \mathbb{N}$ such that $\left(z_{\gamma_{n}}\right)_{n \geq m_{1}} \stackrel{1-\delta}{\sim} l^{1}$ since $z_{\gamma_{n}} \stackrel{\text { alm }}{\sim} l^{1}$. Hence, with the notation $f_{n}=T z_{\gamma_{n}}$, we get $\left(f_{n}\right)_{n \geq m_{1}} \stackrel{1-2 \delta}{\sim} l^{1}$ because

$$
\begin{aligned}
(1-2 \delta) \sum_{n=m_{1}}^{\infty}\left|\alpha_{n}\right| & <(1-\delta)^{2} \sum_{n=m_{1}}^{\infty}\left|\alpha_{n}\right| \leq(1-\delta) \sum_{n=m_{1}}^{\infty} \frac{\left|\alpha_{n}\right|}{\left\|f_{n}\right\|} \\
& \leq\left\|\sum_{n=m_{1}}^{\infty} \frac{\alpha_{n}}{\left\|f_{n}\right\|} z_{\gamma_{n}}\right\| \leq\left\|\sum_{n=m_{1}}^{\infty} \alpha_{n} \frac{f_{n}}{\left\|f_{n}\right\|}\right\| .
\end{aligned}
$$

Then by [5, Th. B] or [24, L. 10,6] there is a sequence $\left(\tilde{f}_{k}\right) \subset l^{1}$ of pairwise disjointly supported elements of $l^{1}$ and a subsequence $\left(f_{n_{k}}\right)$ such that

$$
\left\|f_{n_{k}}-\tilde{f}_{k}\right\|<\delta^{\prime}
$$


where $\delta^{\prime} \rightarrow 0$ as $\delta \rightarrow 0$. Set $\tilde{z}_{k}=T^{-1} \tilde{f}_{k}$. Then

$$
\left\|\tilde{z}_{k}-z_{\gamma_{n_{k}}}\right\|=\left\|T^{-1} \tilde{f}_{k}-T^{-1} f_{n_{k}}\right\| \leq\left\|\tilde{f}_{k}-f_{n_{k}}\right\|<\delta^{\prime} .
$$

Now we choose $\delta>0$ small enough in order to have $\delta^{\prime}\left\|x^{\prime}\right\|<\varepsilon / 4$. Hence

$$
\left|x^{\prime}\left(\tilde{z}_{k}\right)\right| \geq \frac{\varepsilon}{2}-\mid x^{\prime}\left(\tilde{z}_{k}-z_{\left.\gamma_{n_{k}}\right)} \mid \geq \frac{\varepsilon}{2}-\delta^{\prime}\left\|x^{\prime}\right\| \geq \frac{\varepsilon}{4}\right.
$$

for all $k \in \mathbb{N}$. On the other hand, for $e^{\prime}=e_{n_{0}}^{\prime}=\left(T^{-1}\right)^{\prime}\left(x^{\prime}\right)$ we have $e^{\prime}\left(e_{n}\right)=\delta_{n_{0}, n}$ that is

$$
x^{\prime}\left(\tilde{z}_{k}\right)=e^{\prime}\left(\tilde{f}_{k}\right)=0
$$

for all but possibly one $k \in \mathbb{N}$ because the $\tilde{f}_{k}$ are pairwise disjoint. This contradiction proves the Sublemma.

Since the biorthogonal functionals separate $X$ the Sublemma and Theorem 13 imply that $X^{\#}$ is M-embedded and $X=\left(X^{\#}\right)^{\prime}$. The Sublemma states that $\left[x_{n}^{\prime}\right] \subset X^{\#}$. In fact one has $\left[x_{n}^{\prime}\right]=X^{\#}$. To see this let $z^{\prime} \in X^{\#} \backslash\left[x_{n}^{\prime}\right]$. Since $X^{\prime}$ is isomorphic to $l^{\infty}$ there is an infinite set $N^{\prime} \subset \mathbb{N}$ and there is $\varepsilon>0$ such that $\left|z^{\prime}\left(x_{n}\right)\right|>\varepsilon$ for all $n \in N^{\prime}$. But $\left(x_{n}\right)_{n \in N^{\prime}} \stackrel{\text { alm }}{\sim} l^{1}$ whence $\left(x_{n}\right)_{n \in N} \stackrel{\text { asy }}{\sim} l^{1}$ for an appropriate infinite set $N \subset N^{\prime}$ by [23, Cor. 3]. This means that $\left(x_{n}\right)_{n \in N} \tau_{\mu}$-converges to 0 hence $z^{\prime} \notin X^{\#}$.

We have proved that if $X$ is L-embedded and almost isometric to $l^{1}$ then $X$ is the dual of the M-embedded space $X^{\#}$ and $X^{\#}=\left[x_{n}^{\prime}\right]$. To end the proof it just remains to apply Proposition 14 .

The arguments are similar for the case in which $x_{n} \stackrel{\text { asy }}{\sim} l^{1}$.

$\S 5$ On some results of Godefroy, Kalton, $\mathrm{Li}$

First we deal with [10, Prop. 2.1]. The first part of that proposition says that $X^{\#}$ where $X$ is a nicely placed (=L-embedded) subspace of $\mathrm{L}^{1}(\mu)$ is always M-embedded, not only in the situation of Theorem 13 .

Proposition 16 Let $X$ be an L-embedded Banach space. Then $X^{\#}$ is M-embedded.

Proof: With the usual notation $X^{\prime \prime}=X \oplus_{1} X_{s}$ and with Proposition 12 we have

$$
X^{\#}=\left(X_{s}\right)_{\perp} \subset X^{\prime} .
$$

We set

$$
Z={\overline{X_{s}}}^{w^{*}}=\left(X^{\#}\right)^{\perp} \subset X^{\prime \prime}
$$

and

$$
Y=X \cap{\overline{X_{s}}}^{w^{*}}=\left(X^{\#}\right)_{\perp} \subset X .
$$

By Corollary 11, $Y$ is L-embedded because its unit ball

$$
\mathrm{B}_{Y}=\mathrm{B}_{X} \cap \bigcap_{x^{\prime} \in X^{\#}} \operatorname{ker} x^{\prime}
$$

is $\tau_{\mu}$-closed. Hence $Y^{\perp \perp}=Y \oplus_{1}\left(Y^{\perp \perp} \cap X_{s}\right)$ by Lemma 11. Now the fact $Z=Y \oplus_{1} X_{s}$ and the fact that $X^{\#}$ is an M-ideal in its bidual $Y^{\perp}$ can be deduced exactly as in the proof of [10, Prop. 2.1].

For the proof of Proposition 17 we recall property $\left(m_{1}^{*}\right)$. In [16] a separable Banach space $Z$ is defined to have property $\left(m_{1}^{*}\right)$ if for all $z^{\prime}, z_{n}^{\prime} \in Z^{\prime}$

$$
\lim \sup \left\|z^{\prime}+z_{n}^{\prime}\right\|=\left\|z^{\prime}\right\|+\lim \sup \left\|z_{n}^{\prime}\right\|
$$

whenever $z_{n}^{\prime} \stackrel{w^{*}}{\rightarrow} 0$. Analogously a separable Banach space $X$ is defined to have property $\left(m_{1}\right)$ if for all $x, x_{n} \in X$

$$
\lim \sup \left\|x+x_{n}\right\|=\|x\|+\lim \sup \left\|x_{n}\right\|
$$

whenever $x_{n} \stackrel{w}{\rightarrow} 0$.

The second part of [10, Prop. 2.1] reads as follows in our context. 
Proposition 17 Let $X$ be a separable L-embedded Banach space. If $\mathrm{B}_{X}$ is $\tau_{\mu}$-sequentially compact then for any $\varepsilon>0$ there is a subspace $X_{\varepsilon}$ of $c_{0}$ such that $\operatorname{dist}\left(X^{\#}, X_{\varepsilon}\right)<1+\varepsilon$.

Proof: Exactly as in [10] one distinguishes three steps: Firstly one proves that $X^{\#}$ has property $\left(m_{1}^{*}\right)$, secondly one deduces from this property $\left(m_{\infty}^{*}\right)$ and thirdly it remains to apply [16, Th. 3.5]. Only the first step must be modified a bit.

From the proof of Proposition 16 we know that $\left(X^{\#}\right)^{\prime}=X / Y$ where $Y=X \cap \overline{X_{s}} w^{*}=\left(X^{\#}\right)_{\perp} \subset X$. Let $\left(u_{n}\right) \subset X / Y=\left(X^{\#}\right)^{\prime}$ be a $w^{*}$-null sequence. We denote by $Q: X \rightarrow X / Y$ the quotient map. Let $\left(x_{n}\right) \subset X$ be a bounded sequence such that $Q x_{n}=u_{n}$. By hypothesis there is a $\tau_{\mu}$-convergent subsequence - still denoted by $\left(x_{n}\right)$ - such that $x_{n}-x_{0} \stackrel{\text { asy }}{\sim} l^{1}$ where $x_{0}=\tau_{\mu}-\lim x_{n}$ and such that $\lim \left\|x_{0}-x_{n}\right\|$ exists. We have $x_{0} \in Y$ because for any $x^{\prime} \in X^{\#}$ one has

$$
x^{\prime}\left(x_{0}\right)=\lim x^{\prime}\left(x_{m}\right)=\lim x^{\prime}\left(u_{m}\right)=0 .
$$

We have furthermore that

$$
\lim \sup \left\|y+x+\left(x_{n}-x_{0}\right)\right\| \geq\|y+x\|+\lim \left\|x_{n}-x_{0}\right\|
$$

for all $x \in X, y \in Y$. To see this, recall that $X$ is L-embedded, and that by Lemma 2 each universal net $\left(x_{n_{\gamma}}-x_{0}\right) w^{*}$-converges to a limit $x_{s} \in X_{s}$ such that $\left\|x_{s}\right\|=\lim _{\gamma}\left\|x_{n_{\gamma}}-x_{0}\right\|$ and

$$
\lim _{\gamma}\left\|y+x+\left(x_{n_{\gamma}}-x_{0}\right)\right\| \geq\left\|y+x+x_{s}\right\|=\|y+x\|+\left\|x_{s}\right\|
$$

by $w^{*}$-continuity of the norm whence (14).

Since $\|Q x\|=\inf _{y \in Y}\|y+x\|$ we deduce from (14) that

$$
\lim \sup \left\|y+x+\left(x_{n}-x_{0}\right)\right\| \geq\|Q x\|+\lim \left\|u_{n}\right\|
$$

and

$$
\lim \sup \left\|Q x+u_{n}\right\| \geq\|Q x\|+\lim \left\|u_{n}\right\|
$$

which proves that $X^{\#}$ has property $\left(m_{1}^{*}\right)$. The deduction of property $\left(m_{\infty}^{*}\right)$ and the conclusion via [16, Th. $3.5]$ do not depend on the measure topology and coincide therefore with the arguments in [10].

The following remark gives a characterization of property $\left(m_{1}^{*}\right)$ in L-embedded Banach spaces. We will not need it in the sequel and state it only because the way we prove it by constructing asymptotic $l^{1}$-sequences fits naturally in the main theme of this paper. Note that the implication (i) $\Rightarrow$ (ii) holds for arbitrary Banach spaces $Z$, that the implications (i) $\Rightarrow$ (ii) $\Leftrightarrow$ (iii) hold whenever $Z$ is such that its dual admits an abstract measure topology, and that the implication (iii) $\Rightarrow$ (iv) does not need the M-embeddedness of $Z$. Note

furthermore that in Remark 18 the separation assumption on $Z$ could be omitted because the definition of properties $\left(m_{1}^{*}\right)$ and $\left(m_{1}\right)$ makes sense also for non-separable spaces.

\section{Remark 18}

(a) Let $Z$ be a Banach space such that its dual is L-embedded. Then the following assertions are equivalent:

(i) $Z$ has property $\left(m_{1}^{*}\right)$.

(ii) Each $w^{*}$-null sequence in $Z^{\prime}$ admits of a subsequence that converges to 0 in norm or spans $l^{1}$ asymptotically.

(iii) Each $w^{*}$-null sequence in $Z^{\prime}$ is $\tau_{\mu}$-null.

If $Z$ is even separable and $M$-embedded then the assertions above are equivalent to

(iv) $\mathrm{B}_{Z^{\prime}}$ is $\tau_{\mu}$-sequentially compact.

(b) An arbitrary Banach space $X$ has property $\left(m_{1}\right)$ if and only if it has the Schur property. 
Proof: (a) We set $X=Z^{\prime}$. Sketch of

(i) $\Rightarrow\left(\right.$ ii): The proof ressembles the one of [23, Th. 2] the only difference being that (12) replaces the $w^{*}$-lower semicontinuity of the norm and the L-embeddedness of $Z^{\prime}$. Let $\left(x_{m}\right) \subset X$ be a $w^{*}$-null sequence, suppose without loss of generality that $\left\|x_{m}\right\|=1$. Let $\left(\delta_{n}\right)$ be a sequence of strictly positive numbers converging to 0 . Set $\eta_{1}=\frac{1}{6} \delta_{1}$ and $\eta_{n+1}=\frac{1}{6} \min \left(\eta_{n}, \delta_{n+1}\right)$ for $n \in \mathbb{N}$. By induction over $n \in \mathbb{N}$ one constructs $m_{n} \in \Gamma$ such that

$$
\left(\sum_{i=1}^{n}\left(1-\delta_{i}\right)\left|\alpha_{i}\right|\right)+\eta_{n} \sum_{i=1}^{n}\left|\alpha_{i}\right| \leq\left\|\sum_{i=1}^{n} \alpha_{i} x_{m_{i}}\right\| \leq \sum_{i=1}^{n}\left|\alpha_{i}\right| \quad \forall n \in \mathbb{N}, \alpha_{i} \in \mathbb{C} .
$$

For the induction step $n \mapsto n+1$ fix an element $\alpha=\left(\alpha_{i}\right)_{i=1}^{n+1}$ in the unit sphere of $l_{n+1}^{1}$ such that $\alpha_{n+1} \neq 0$. Then (12) yields

$$
\begin{aligned}
& \left\|\left(\sum_{i=1}^{n} \alpha_{i} x_{m_{i}}\right)+\alpha_{n+1} x_{m}\right\|+\frac{1}{2} \min \left(\eta_{n}, \delta_{n+1}\right) \\
& \geq\left\|\sum_{i=1}^{n} \alpha_{i} x_{m_{i}}\right\|+\left|\alpha_{n+1}\right| \\
& \stackrel{\text { 1.5) }}{\geq}\left(\sum_{i=1}^{n}\left(1-\delta_{i}\right)\left|\alpha_{i}\right|\right)+\eta_{n}\left(\sum_{i=1}^{n}\left|\alpha_{i}\right|\right)+\left|\alpha_{n+1}\right| \\
& \geq\left(\sum_{i=1}^{n+1}\left(1-\delta_{i}\right)\left|\alpha_{i}\right|\right)+\min \left(\eta_{n}, \delta_{n+1}\right)
\end{aligned}
$$

whence

$$
\left\|\left(\sum_{i=1}^{n} \alpha_{i} y_{m_{i}}^{(i)}\right)+\alpha_{n+1} y_{m}^{(n+1)}\right\| \geq\left(\sum_{i=1}^{n+1}\left(1-\delta_{i}\right)\left|\alpha_{i}\right|\right)+3 \eta_{n+1}
$$

for infinitely many $m$. This gives (15); the details are the same as in the proof of [23, Th. 2] or of Lemma 6 . Note for the proof of part (b) below that we used the $w^{*}$-convergence of $\left(x_{n}\right)$ only in order to apply property $\left(m_{1}^{*}\right)$ but not for the construction of the $l^{1}$-basis itself.

(ii) $\Rightarrow$ (i): Suppose that $Z$ satisfies (ii) without having $\left(m_{1}^{*}\right)$ and suppose that $Z^{\prime}$ is L-embedded. Then there are $x \in X, \varepsilon>0$ and a $w^{*}$-null sequence $\left(x_{n}\right) \subset X$ such that $\lim \left\|x+x_{n}\right\|$ and $\lim \left\|x_{n}\right\|$ exist and

$$
\varepsilon+\lim \left\|x+x_{n}\right\|<\|x\|+\lim \left\|x_{n}\right\| .
$$

Since (16) excludes the case $\left\|x_{n}\right\| \rightarrow 0$ we have - after passing to an appropriate subsequence - that $x_{n} \stackrel{\text { asy }}{\sim} l^{1}$. Let $\left(x_{n_{\gamma}}\right)$ be a universal net. By Lemma 2 it $w^{*}$-converges to a point $x_{s} \in X_{s}$ and

$$
\lim _{\gamma}\left\|x+x_{n_{\gamma}}\right\| \geq\left\|x+x_{s}\right\|=\|x\|+\lim _{\gamma}\left\|x_{n_{\gamma}}\right\|
$$

by $w^{*}$-continuity of the norm which contradicts $(16)$ and proves property $\left(m_{1}^{*}\right)$.

(ii) $\Leftrightarrow$ (iii) is immediate from the definition of $\tau_{\mu}$.

(iii) $\Rightarrow$ (iv): If $Z$ is separable then $\mathrm{B}_{X}$ is $w^{*}$-sequentially compact. Hence $\mathrm{B}_{X}$ is $\tau_{\mu}$-sequentially compact if (iii) holds.

(iv) $\Rightarrow$ (i): If $Z$ is M-embedded then $Z=X^{\#}$. In this case, if $\mathrm{B}_{X}$ is $\tau_{\mu}$-sequentially compact then the proof of Proposition 17 shows that $Z$ has $\left(m_{1}^{*}\right)$.

(b) The Schur property clearly implies $\left(m_{1}\right)$. Conversely, suppose a Banach space $X$ has $\left(m_{1}\right)$ but fails to have the Schur property. Then there exists a normalized $w$-null sequence $\left(x_{n}\right)$ in $X$. Exactly as in (i) $\Rightarrow($ ii) of part (a), using (13) instead of (12), we can extract a subsequence $\left(x_{n_{k}}\right)$ which spans $l^{1}$ asymptotically. But a normalized sequence spanning $l^{1}$ cannot converge weakly. This contradiction proves that $\left(m_{1}\right)$ implies the Schur property.

Sublemma 19 For any L-embedded space $X$ one has the following inclusions

$$
\begin{aligned}
X \cap{\overline{\mathrm{B}_{X_{s}}}}^{w^{*}} & \subset \bigcap\left\{\bar{V}^{w} \mid V \tau_{\mu}-\text { open in } \mathrm{B}_{X}, 0 \in V\right\} \\
& \subset \bigcap\left\{\overline{c o}^{\|\cdot\|}(V) \mid V \tau_{\mu}-\text { open in } \mathrm{B}_{X}, 0 \in V\right\} .
\end{aligned}
$$


Proof: The first inclusion is immediate from (i) of Lemma 5, the second inclusion follows from $\overline{\mathrm{co}}^{w}(V)=$ $\overline{\mathrm{co}}\|\cdot\|(V)$.

That at the time of this writing we are not able to give the whole analogue of [10, L. 2.6] is essentially due to the fact that we know only the behaviour of sequences but not of nets in $\tau_{\mu}$, more specifically if we knew that one can extract a $\tau_{\mu}$-convergent sequence from a $\tau_{\mu}$-convergent net then we could also prove that $\bigcap \overline{\mathrm{co}}\|\cdot\|(V) \subset X \cap \overline{\mathrm{B}}_{X_{s}}{ }^{*}$. In any case, our reduced version suffices for Lemma 20 which corresponds to 10 , L. 2.7]. Note that (ii) of Lemma 20 is equivalent to the two conditions of Theorem 13 .

Lemma 20 Let $X$ be an L-embedded Banach space. The following assertions are equivalent.

(i) There exists a locally convex Hausdorff topology on $X$ which is coarser than $\tau_{\mu}$ on $\mathrm{B}_{X}$.

(ii) $\mathrm{B}_{X}$ is compact Hausdorff with respect to $\sigma\left(X, X^{\#}\right)$.

(iii) $\{0\}$ is the intersection of the convex $\tau_{\mu}$-neighbourhoods of 0 in $\mathrm{B}_{X}$.

If moreover $\mathrm{B}_{X}$ is $\tau_{\mu}$-compact Hausdorff, then the above conditions are also equivalent to

(iv) The weak topology of $X$ is finer than $\tau_{\mu}$ on $\mathrm{B}_{X}$.

(v) There exists a locally convex Hausdorff topology - namely $\sigma\left(X, X^{\#}\right)$ - which coincides with $\tau_{\mu}$ on $\mathrm{B}_{X}$.

(vi) 0 has a basis of $\tau_{\mu}$-neighbourhoods in $\mathrm{B}_{X}$ consisting of convex sets.

Proof: The proof is word-for-word the same as the one of [10, L. 2.7] (with Sublemma 19 replacing [10, L. $2.6])$.

Suppose that with the notation of (i) of Lemma 5 one has $x=x^{\prime \prime} \in X$; then $x_{\gamma} \stackrel{\tau_{\mu}}{\rightarrow} 0$ and $x_{\gamma} \stackrel{w}{\rightarrow} x$. If (iv) of Lemma 20 holds then this means that both $x_{\gamma} \stackrel{\tau_{\mu}}{\rightarrow} 0$ and $x_{\gamma} \stackrel{\tau_{\mu}}{\rightarrow} x$. Since it is not clear whether $\tau_{\mu}$ is Hausdorff we cannot deduce that $x=0$. Therefore we claimed $\mathrm{B}_{X}$ to be $\tau_{\mu}$-Hausdorff for (iv) - (vi) in Lemma 20 (in particular for the proof of (iv) $\Rightarrow($ ii)).

We end this section with some remarks on the continuity of the canonical L-projection $P$ on the bidual of an L-embedded space $X$.

In 10, Prop. 3.8] it was shown that $P$ is $\left(w^{*}-\tau_{\mu}\right)$-sequentially continuous if and only if $X$ has the Schur property. In the proof the authors of [10] use the Grothendieck property of $\mathrm{L}^{\infty}$ in order to obtain the $\left(w^{*}-w\right)$-sequential continuity of the L-projection on $\left(\mathrm{L}^{\infty}\right)^{\prime}$. But in the general case it is not known whether $P$ is always $\left(w^{*}-w\right)$-sequentially continuous although by an example of Johnson it is known that in general the dual of an L-embedded Banach space does not have the Grothendieck property. Therefore it is not clear whether the first part of [10, Prop. 3.8] can be generalized in our context; its second part can:

Proposition 21 Let $X$ be L-embedded and $P$ be the canonical L-projection on $X^{\prime \prime}$. Suppose that the abstract measure topology $\tau_{\mu}$ on $X$ is Hausdorff. Then $P$ is $\left(w^{*}-\tau_{\mu}\right)$-continuous if and only if the restriction of $\tau_{\mu}$ to $\mathrm{B}_{X}$ is compact Hausdorff locally convex.

Proof: See [10, Prop. 3.8(b)].

$\S 6$ The special case of the predual of a von Neumann algebra

Recall that two elements $\phi, \psi \in \mathcal{N}_{*}$ in the predual of a von Neumann algebra $\mathcal{N}$ are called orthogonal - $\phi \perp \psi$ in symbols - if they have orthogonal right and orthogonal left support projections. It is well know that $\phi \perp \psi$ if and only if the linear span of $\phi$ and $\psi$ is isometrically isomorphic to the two-dimensional $l^{1}(2)$.

Sublemma 22 Let $\mathcal{N}$ be a von Neumann algebra with predual $X=\mathcal{N}_{*}$. For every $\varepsilon>0$ there is $\delta>0$ with the following property.

If $x, y, z \in \mathrm{B}_{X}$ are such that

$$
\begin{aligned}
& \left\|\alpha \frac{z}{\|z\|}+\beta \frac{x}{\|x\|}\right\| \geq(1-\delta)(|\alpha|+|\beta|) \\
& \left\|\alpha \frac{z}{\|z\|}+\beta \frac{y}{\|y\|}\right\| \geq(1-\delta)(|\alpha|+|\beta|)
\end{aligned}
$$


then there are $\tilde{x}, \tilde{y}, \tilde{z} \in X$ of norm one such that

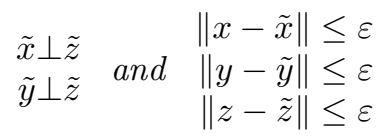

Proof: We have already recalled the fact that two normal functionals on a von Neumann algebra are orthogonal if and only if they span $l^{1}(2)$ isometrically. A second ingredient of this proof is the fact that the ultraproduct of a family of preduals of von Neumann algebras is again a predual of a von Neumann algebra [12], 27]. It is now enough to combine these two facts with a standard ultraproduct argument [14].

It is well known that if $\mathcal{N}$ is a von Neumann algebra with a finite faithful normal trace $\tau$ then the measure topology on the predual $\mathcal{N}_{*}$ defined via $\tau$ is metrizable and makes $\mathcal{N}_{*}$ a Hausdorff topological vector space. For the abstract measure topology $\tau_{\mu}$ on the predual of an arbitrary von Neumann algebra we only know by the following lemma (and by Lemmas (5, 6) that multiplication by scalars and addition are continuous and that on bounded sets $\tau_{\mu}$ makes the von Neumann predual a Fréchet space.

Proposition 23 Let $X=\mathcal{N}_{*}$ be the predual of a von Neumann algebra $\mathcal{N}$. If $X$ is endowed with the abstract measure toplogy $\tau_{\mu}$ which it has by Theorem 6 then addition is $\tau_{\mu}$-continuous.

Proof: Let $\left(x_{n}\right),\left(y_{n}\right)$ be two sequences in $\mathrm{B}_{X}$ each of which spans $l^{1}$ asymptotically. Suppose there is $\varepsilon>0$ such that $\left\|z_{n}\right\|>\varepsilon$ where $z_{n}=x_{n}+y_{n}$, suppose further that $\lim \left\|x_{n}\right\|, \lim \left\|y_{n}\right\|, \lim \left\|z_{n}\right\|$ exist. To show the Proposition it is enough to show that there is a subsequence $\left(z_{n_{k}}\right)$ spanning $l^{1}$ asymptotically.

Let $\left(\delta_{n}\right)$ be a sequence of strictly positive numbers in ]0,1] converging to 0 . Set $\eta_{1}=\frac{1}{3} \delta_{1}$ and $\eta_{n+1}=$ $\frac{1}{3} \min \left(\eta_{n}, \delta_{n+1}\right)$ for $n \in \mathbb{N}$. By induction over $m \in \mathbb{N}$ we construct a sequence $\left(n_{m}\right)$ such that

$$
\left\|\sum_{k=1}^{m} \alpha_{k} \frac{z_{n_{k}}}{\left\|z_{n_{k}}\right\|}\right\| \geq\left(\sum_{k=1}^{m}\left(1-\delta_{k}\right)\left|\alpha_{k}\right|\right)+\eta_{m} \sum_{k=1}^{m}\left|\alpha_{k}\right|
$$

for all integers $m$ and all scalars $\alpha_{k}$. Since the construction ressembles the proofs of [23, Th. 2], of Lemma 6, and of Remark 18 we detail it only for $m=2$.

Let $\theta_{2}>0$ be arbitrary for the moment and to be determined later. Set $n_{1}=1$.

ClaIM: There is $n_{2} \in \mathbb{N}$ such that

$$
\begin{aligned}
& \left\|\alpha \frac{z_{1}}{\left\|z_{1}\right\|}+\beta \frac{x_{n_{2}}}{\left\|x_{n_{2}}\right\|}\right\| \geq\left(1-\theta_{2}\right)(|\alpha|+|\beta|) \\
& \left\|\alpha \frac{z_{1}}{\left\|z_{1}\right\|}+\beta \frac{y_{n_{2}}}{\left\|y_{n_{2}}\right\|}\right\| \geq\left(1-\theta_{2}\right)(|\alpha|+|\beta|)
\end{aligned}
$$

for all scalars $\alpha, \beta$. (As usual in this paper) this is essentially due to the $w^{*}$-lower semicontinuity of the norm by which one has $\liminf \inf _{\gamma}\left\|\alpha \frac{z_{1}}{\left\|z_{1}\right\|}+\beta \frac{x_{n_{\gamma}}}{\left\|x_{n_{\gamma}}\right\|}\right\| \geq\left\|\alpha \frac{z_{1}}{\left\|z_{1}\right\|}+\beta \frac{x_{s}}{\left\|x_{s}\right\|}\right\|=|\alpha|+|\beta|$ where $x_{s} \in X_{s}$ is the $w^{*}$-limit of a universal net $\left(x_{n_{\gamma}}\right)$ and $\left\|x_{s}\right\|=\lim _{\gamma}\left\|x_{n_{\gamma}}\right\|$ (see Lemma 2); hence there are infinitely many $n_{2}$ satisfying (18). Applying the same reasoning to the corresponding subsequence of $\left(y_{n}\right)$ gives the CLAim.

By Sublemma 22 there are $\tilde{x}_{2}, \tilde{y}_{2}, \tilde{z}_{1}$ in $X$ such that

$$
\left\|x_{n_{2}}-\tilde{x}_{2}\right\| \leq \theta_{2}^{\prime},\left\|y_{n_{2}}-\tilde{y}_{2}\right\| \leq \theta_{2}^{\prime},\left\|z_{1}-\tilde{z}_{1}\right\| \leq \theta_{2}^{\prime},
$$

and

$$
\tilde{z}_{1} \perp \tilde{x}_{2}, \quad \tilde{z}_{1} \perp \tilde{y}_{2}
$$

where $\theta_{2}^{\prime} \rightarrow 0$ as $\theta_{2} \rightarrow 0$. Now (20) implies that

$$
\tilde{z}_{1} \perp \tilde{z}_{n_{2}}
$$

where $\tilde{z}_{n_{2}}=\tilde{x}_{n_{2}}+\tilde{y}_{n_{2}}$ which means that $\left\|\alpha \frac{\tilde{z}_{1}}{\left\|\tilde{z}_{1}\right\|}+\beta \frac{\tilde{z}_{2}}{\left\|\tilde{z}_{2}\right\|}\right\|=|\alpha|+|\beta|$. Hence, if $\theta_{2}$ is choosen small enough, we get (17) for $m=2$. It is now left to the reader to iterate the construction in order to end the induction and thus the proof.

As already noticed in the remarks concerning the questions after Corollary 9, Randrianantoanina [26] has proved that the Kadec-Pełczyński splitting lemma holds for preduals of von Neumann algebras. With this result Komlos' theorem follows almost immediately for von Neumann preduals. 
Proposition 24 (Komlos) Each bounded sequence in the predual of a von Neumann algebra admits a subsequence whose Cesaro means converge with respect to the abstract measure topology.

Proof: Let $X=\mathcal{N}_{*}$ be the predual of a von Neumann algebra $\mathcal{N}$. Endow $X$ with its abstract measure topology $\tau_{\mu}$. Let $\left(x_{n}\right) \subset X$ be bounded. Then by [26] there is a subsequence $\left(x_{n_{k}}\right)$ and there is a decomposition $x_{n_{k}}=y_{k}+z_{k}$ where $\left(z_{k}\right) w$-converges and such that there is a sequence $\left(\tilde{y}_{k}\right)$ of pairwise orthogonal elements in $X$ with

$$
\left\|\tilde{y}_{k}-y_{k}\right\| \rightarrow 0 \text {. }
$$

If one applies the classical theorem of Komlos to the isometric $l^{1}$-copy $\left[\tilde{y}_{k}\right]$ then one obtains, after passing to an appropriate subsequence, that the Cesaro means of any subsequence of $\left(\tilde{y}_{k}\right)$ converge with respect to the measure (=pointwise) topology of $\left[\tilde{y}_{k}\right]$ whence with respect to $\tau_{\mu}$. So do the Cesaro means $\hat{y}_{k}$ of the $y_{k}$ by (21). It is known that von Neumann preduals have the weak Banach-Saks property [2]. Hence, again after passing to the appropriate subsequences of $\left(y_{k}\right)$ and $\left(z_{k}\right)$, the Cesaro means $\hat{z}_{k}$ of the $z_{k}$ converge in norm whence with respect to $\tau_{\mu}$. Since $\hat{x}_{k}=\hat{y}_{k}+\hat{z}_{k}$ where $\hat{x}_{k}$ denote the Cesaro means of the $x_{n_{k}}$, the assertion now follows from Proposition 23.

ACKNowledgement I thank Dirk Werner for several helpful discussions.

\section{References}

[1] B. Beauzamy. Banach-Saks properties and spreading models. Math. Scand., 44:357-384, 1979.

[2] A. Belanger and J. Diestel. A remark on weak convergence in the dual of a $\mathrm{C}^{*}$-algebra. Proc. Amer. Math. Soc., 98:185-186, 1986.

[3] A. V. Buhvalov and G. J. Lozanovskii. On sets closed in measure in spaces of measurable functions. Trans. Moscow Math. Soc., 2:127-148, 1978.

[4] J. Diestel. Sequences and Series in Banach Spaces. Springer, Berlin-Heidelberg-New York, 1984.

[5] L. E. Dor. On projections in $L_{1}$. Ann. of Math., 102:463-474, 1975.

[6] P. N. Dowling, W. B. Johnson, C. J. Lennard, and B. Turett. The optimality of James's distortion theorems. Proc. Amer. Math. Soc., 125:167-174, 1997.

[7] N. Dunford and J. T. Schwartz. Linear Operators. Part 1: General Theory. Interscience Publishers, New York, 1958.

[8] R. Engelking. General Topology. Heldermann Verlag, Berlin, 1989.

[9] G. Godefroy. Sous-espaces bien disposés de $L^{1}$ - Applications. Trans. Amer. Math. Soc., 286:227-249, 1984.

[10] G. Godefroy, N. Kalton, and D. Li. On subspaces of $L^{1}$ which embed into $l_{1}$. J. Reine Angew. Math., 471:43-75, 1996.

[11] G. Godefroy and D. Li. Some natural families of M-ideals. Math. Scand., 66:249-263, 1990.

[12] U. Groh. Uniform ergodic theorems for identity preserving Schwartz maps on $W^{*}$-algebras. J. Operator Theory, 11:395-404, 1984.

[13] P. Harmand, D. Werner, and W. Werner. M-ideals in Banach Spaces and Banach Algebras. Lecture Notes in Mathematics 1547. Springer, 1993.

[14] S. Heinrich. Ultraproducts in Banach space theory. J. Reine Angew. Math., 313:72-104, 1980.

[15] I. Kadec and A. Pełczyński. Bases, lacunary sequences and complemented subspaces in the space $\mathrm{L}_{p}$. Studia Math., 21:161-176, 1962.

[16] N. Kalton and D. Werner. Property $(M), M$-ideals, and almost isometric structure of Banach spaces. J. Reine Angew. Math., 461:137-178, 1995. 
[17] J. L. Kelley. General Topology. D. van Nostrand Company, 1963.

[18] J. Kisyński. Convergence du type L. Colloquium Mathematicum, 7:205-211, 1960.

[19] D. Li. Espaces $L$-facteurs de leurs biduaux: bonne disposition, meilleure approximation et propriété de Radon-Nikodym. Quart. J. Math. Oxford (2), 38:229-243, 1987.

[20] J. Lindenstrauss and L. Tzafriri. Classical Banach Spaces I. Springer, Berlin-Heidelberg-New York, 1977.

[21] J. Lindenstrauss and L. Tzafriri. Classical Banach Spaces II. Springer, Berlin-Heidelberg-New York, 1979.

[22] G. K. Pedersen. C $C^{*}$-algebras and Their Automorphism Groups. Academic Press, London, New York, San Francisco, 1979.

[23] H. Pfitzner. A note on asymptotically isometric copies of $l^{1}$ and $c_{0}$. Proc. Amer. Math. Soc., 2000. to appear.

[24] H. Pfitzner. Perturbations of $l^{1}$-copies and convergence in preduals of von Neumann algebras. $J$. Operator Theory, 2000. to appear.

[25] G. Pisier. Bases, suites lacunaires dans les espaces $\mathrm{L}_{p}$ d'après Kadec et Pelczynski. Séminaire MaureySchwartz de l'Ecole Polytechnique, pages Exposés 18 - 19, 1972-73.

[26] N. Randrianantoanina. Kadec-Pełczyński decomposition for Haagerup $L^{p}$-spaces. Preprint, 2000.

[27] Yves Raynaud. On ultrapowers of non commutative $L_{p}$ spaces. Preprint, 2000.

[28] M. Takesaki. Theory of Operator Algebras I. Springer, Berlin-Heidelberg-New York, 1979.

Hermann Pfitzner

Université d'Orléans

BP 6759

F-45067 Orléans Cedex 2

France

e-mail:pfitzner@labomath.univ-orleans.fr 